\title{
Coupling of meshfree methods with finite elements: Basic concepts and test results
}

\author{
T.Rabczuk $k^{+}$, S.P.Xiao ${ }^{\dagger}$, M.Sauer $\ddagger$ \\ Institute for Numerical Mechanics, University of Munich, Germany
}

\begin{abstract}
SUMMARY
This paper reviews several novel and older methods for coupling meshfree particle methods, particularly the elementfree Galerkin (EFG) method and the Smooth Particle Hydrodynamics (SPH), with finite elements. We study master slave couplings where particles are fixed across the finite element boundary, coupling via interface shape functions such that consistency conditions are satisfied, bridging domain coupling, compatibility coupling with Lagrange multipliers and hybrid coupling methods where forces from the particles are applied via their shape functions on the FE nodes and vice versa. The hybrid coupling methods are well suited for large deformations and adaptivity and the coupling procedure is independent from the particle distance and nodal arrangement. We will study the methods for several static and dynamic applications, compare the results to analytical and experimental data and show advantages and drawbacks of the methods. Copyright (c) 2000 John Wiley \& Sons, Ltd.
\end{abstract}

KEY WORDS: meshfree particle methods; adaptivity; EFG; SPH

\section{INTRODUCTION}

Meshfree methods became a good alternative to FEM and FDM in certain areas. For problems involving large deformation, fracture and fragmentation, meshfree methods seem to be more flexible than finite elements because they do not rely on a fixed topological connectivity between nodes. Meshfree methods have advantages in problems involving crack growth as no remeshing is necessary. Particles can be added without the need to remesh, so adaptive refinement of the discretization can be done easily.

Considerable research in meshfree methods has been devoted on inherent difficulties like consistency, stability and Dirichlet boundary conditions. While these issues are not yet completely resolved, viable methods are available. In addition, up to now, the computational

\footnotetext{
* Correspondence to: $\dagger$ Assistant Professor, Department of Mechanical and Industrial Engineering, University of Iowa

+ Research Fellow, Institute for Numerical Mechanics, University of Munich, Germany

$\ddagger$ Fraunhofer Institute for Short Time Dynamics, Ernst-Mach-Institute, Eckerstrasse 4, 79106 Freiburg, Germany

Contract/grant sponsor: Publishing Arts Research Council; contract/grant number: 98-1846389
} 
effort for meshfree methods is higher than for finite elements. Hence, as long as no robust and, at the same time, efficient formulation for meshfree particle methods is available, it seems beneficial to discretize only certain parts of the domain with particles and the rest with finite elements. Within this article, we will review some selected coupling approaches in detail. A good overview about i.a. coupling methods can be found in the book of Li and Liu [32].

One of the first coupling procedures for meshfree particle methods and finite elements was proposed by Attaway et al. [2]. They developed a master-slave coupling for fluid structure interactions; the fluid was discretized with particles, the structure was modelled with finite elements. Their algorithm is based on a common master slave coupling (see Belytschko et al. [10]); in every time step they checked whether particles penetrate element faces. The calculated forces that prevent the interpenetration are always normal to the corresponding element surface. Sliding between particles and elements in tangential direction is allowed. A similar approach was proposed by Johnson [24] and Johnson et al. [25]. In addition, they developed transition elements where particles are fixed to FE nodes. This allows for a rigid coupling in the sense that tensile and shear forces are transferred through the interface. We will propose here a new approach where the particles are rigidly fixed to the FE nodes via a master-slave coupling as described in Belytschko et al. [10].

Liu et al. $[33,34]$ showed how to couple the reproducing kernel particle method (RKPM) with FEM by modifying the shape functions in the transition area for both RKPM and FEM. They applied the reproducing condition also in the transition area. Belytschko et al. [4] developed a coupling algorithm for EFG and FEM by a mixed interpolation in the transition domain, where FE nodes are substituted by particles and connected via ramp functions to the EFG nodes so that continuity and consistency are preserved on the interface elements. In [5] they extended this method also for a nodal integration of EFG. The drawback of this method is that the derivatives are discontinuous along the interface.

Huerta et al. [22, 23] developed a mixed hierarchical approximation based on finite elements and meshfree methods. They enriched both FE and EFG method and were able to remove the discontinuities in the derivative across the interior boundaries that was the major drawback of the method in [4].

Hegen [21] coupled FEM and EFG with Lagrange multipliers for elasto-static problems. In his approach, the substitution of FE nodes by particles is not necessary. Rabczuk and Belytschko [44] extended this idea to nonlinear problems and applied it to deformable interfaces. Karutz [28] showed convergence of a similar approach to model crack propagation problems using an adaptively generated EFG domain. A coupling with Lagrange multipliers where finite elements and particles overlap was invented by Belytschko et al. [6]. They called this method bridging domain coupling method and successfully applied it to atomic and multiscale simulations. Another bridging domain coupling was before proposed by Wagner and Liu [49] to couple atomic and continuum simulations, see also Liu et al. [40] for a more comprehensive overview. While the bridging domain coupling in [6] suffered from spurious wave reflections in certain cases, Kadowaki and Liu [26, 27] introduced some wave reflection algorithms to remove this drawback.

Sauer [45] proposed an SPH-FE coupling by extending the SPH domain onto the FE mesh. Different possibilities for exchanging forces between finite element nodes and particles were shown, and the approach was used for adaptive conversion of elements into particles. The main differences to most above mentioned methods is that they used a strong - form coupling.

Recently, the group around Prof. W.K. Liu and S. Li [39, 30, 46] developed a hybrid 
method called reproducing kernel element method (RKEM) which exploits advantages of both, meshfree and finite element methods, e.g. the RKEM shape functions fulfill the Kronecker delta property. A similar method (moving particle finite element method) was developed almost simultaneously by Hao et al. [20]. While most hybrid FE-meshfree methods are at least first order in convergence, Liu et al. [39] showed that their RKEM method maintains the usual convergence rate. Another method to maintain the usual convergence rate by hierarchical enrichment was proposed by Wagner and Liu [48] and Han et al. [18]. Other good overviews about meshfree and particle methods, their coupling to finite elements with impressive examples can be found in $\mathrm{Li}$ and $\mathrm{Liu}[31,32]$.

This article is arranged as follows. We will briefly review the EFG- and SPH-method and finite element shape function. Then we will describe the coupling approaches tested in our article, i.e. master slave couplings, coupling via mixed interpolation, coupling via Lagrange multipliers, bridging domain coupling and hybrid couplings. We will compare these methods for two examples where an analytical solution is available: in statics, the cantilever beam and in dynamics, a rod with initial boundary conditions. The last example was studied by Rabczuk et al. [43] for different meshfree methods. We will further apply the methods to several static and dynamic problems where experimental data is available and discuss some advantages and drawbacks of the different methods.

\section{BASIC EQUATIONS}

The basic equations of continuum mechanics in a total Lagrangian description are the conservation of mass

$$
\varrho J=\varrho_{0} J_{0}
$$

the conservation of linear momentum

$$
\ddot{\mathbf{u}}=\frac{1}{\varrho_{0}} \nabla \cdot \mathbf{P}+\mathbf{b}
$$

and the conservation of angular momentum

$$
\mathbf{F} \cdot \mathbf{P}=\mathbf{P}^{T} \cdot \mathbf{F}^{T}
$$

where $J$ and $J_{0}$ is the Jacobian and initial Jacobian, $\mathbf{u}$ is the displacement vector, $\varrho_{0}$ is the initial density, $\mathbf{P}$ are the nominal stresses, $\mathbf{b}$ are the body forces and $\mathbf{F}$ denotes the deformation gradient. The superimposed dot indicates the material time derivatives and the superscript $T$ denotes the transpose of a tensor or a vector.

The boundary conditions are:

$$
\begin{gathered}
\mathbf{u}(\mathbf{X}, t)=\overline{\mathbf{u}}(\mathbf{X}, t) \quad \text { on } \quad \Gamma_{0}^{u} \\
\mathbf{n}^{0} \cdot \mathbf{P}(\mathbf{X}, t)=\overline{\mathbf{t}}(\mathbf{X}, t) \quad \text { on } \quad \Gamma_{0}^{t}
\end{gathered}
$$

where $\overline{\mathbf{u}}$ and $\overline{\mathbf{t}}$ are the prescribed displacement and traction, respectively, $\mathbf{n}^{0}$ is the outward normal to the domain in the initial configuration and $\Gamma_{0}^{u} \cup \Gamma_{0}^{t}=\Gamma_{0}, \Gamma_{0}^{u} \bigcap \Gamma_{0}^{t}=0$. 


\section{SHAPE FUNCTIONS}

In any of the approximation methods we consider the approximation of the trial functions by

$$
\mathbf{u}(\mathbf{X}, t)=\sum_{I=1}^{N} N_{I}(\mathbf{X}) \mathbf{u}_{I}(t)
$$

where $N_{I}(\mathbf{X})$ is a shape function and $\mathbf{u}_{I}$ is a parameter associated with node $\mathbf{X}_{I}$. For interpolants, such as FE shape functions $\mathbf{u}\left(\mathbf{X}_{I}, t\right)=\mathbf{u}_{I}(t)$. The test functions are approximated by

$$
\delta \mathbf{u}(\mathbf{X})=\sum_{I=1}^{N} N_{I}(\mathbf{X}) \delta \mathbf{u}_{I}
$$

We have chosen the same shape functions for the approximation of the test and trial functions.

\subsection{FE shape functions}

Within this paper, we restrict ourselves to two-dimensional problems. The standard bilinear shape functions for two dimensional four-node finite elements are

$$
N_{I}(\xi)=1 / 4\left(1+\xi_{I} \xi\right)\left(1+\eta_{I} \eta\right)
$$

where $\mathbf{X}(\boldsymbol{\xi})$ is a mapping from the parent domain of problem to the physical domain and $\xi_{I}$ and $\eta_{I}$ are the nodal coordinates in the parent domain $\xi \in[-1,1], \eta \in[-1,1]$, given in

$$
\mathbf{X}=\sum_{I=1}^{N} \mathbf{X}_{I} N_{I}
$$

\subsection{Meshfree shape functions}

In the literature there exists a large amount of meshfree particle methods. As alreday mentioned, we consider the EFG- and SPH-method $([7,8,9,16,41])$ although the coupling ideas presented in this paper are in general applicable to most meshfree methods. A Lagrangian kernel is chosen, i.e. the shape functions are evaluated in the reference configuration only.

3.2.1. SPH shape functions In standard $\mathrm{SPH}$, the shape function is a product of particle volume and a weighting function:

$$
N(\mathbf{X})=\Delta V_{I} W\left(\mathbf{X}-\mathbf{X}_{I}, h_{0}\right)
$$

where $\Delta V_{I}$ is the volume associated with the neighbor particle and $W\left(\mathbf{X}-\mathbf{X}_{I}, h_{0}\right)$ is a weighting function.

3.2.2. EFG shape functions The EFG shape functions are given by

$$
N(\mathbf{X})=\mathbf{p}(\mathbf{X}) \cdot A(\mathbf{X})^{-1} \cdot \mathbf{p}_{j} W\left(\mathbf{X}-\mathbf{X}_{I}, h_{0}\right)
$$

with 


$$
A(\mathbf{X})=\sum_{I=1}^{N} p_{j} p_{j}^{T} W\left(\mathbf{X}-\mathbf{X}_{I}, h_{0}\right)
$$

where $\mathbf{p}(\mathbf{X})$ are basis functions which are chosen to $\mathbf{p}(\mathbf{X})=\left(\begin{array}{llll}1 & X & Y & Z\end{array}\right)$ to fulfill linear completeness (see Belytschko et al. [5]). When the base functions are chosen to $p(\mathbf{X})=(1)$, they are known as Shepard functions and denoted by $w$ in the following.

\section{QUADRATURE FOR FINITE ELEMENT AND PARTICLE METHODS}

For the FEM method, the integrals are customarily evaluated by Gauss quadrature:

$$
\int_{\Omega_{0}} u(\mathbf{X}) d \Omega_{0}=\int_{-1}^{+1} \int_{-1}^{+1} u(\boldsymbol{\xi}) \frac{1}{8} \operatorname{det} \mathbf{J}(\boldsymbol{\xi}) d \boldsymbol{\xi}=\sum_{J=1}^{N_{G P}} w_{P} u_{p}(\boldsymbol{\xi}) \frac{1}{8} \operatorname{det} \mathbf{J}_{p}(\boldsymbol{\xi})
$$

where $\boldsymbol{\xi}$ indicates the coordinates of the local element coordinate system, $\mathbf{J}$ is the Jacobian determinant and $w_{p}$ are the weighting coefficients which depend on the number of Gauss points in the cell. The Jacobian matrix $\mathbf{J}=\partial \mathbf{x} / \partial \boldsymbol{\xi}$ follows from 9

$$
\mathbf{J}=\sum_{J=1}^{N_{G P}} \frac{\partial N_{J}(\boldsymbol{\xi})}{\partial \boldsymbol{\xi}} \mathbf{x}_{J}
$$

The bilinear shape functions mentioned in eq. (8) can be integrated exactly using a $2 \times 2$ quadrature. In this work, however, we use the common reduced integration scheme with only one integration point in the element center which is computational cheaper.

For the particle methods we use an integration by stress points, see e.g. Belytschko et al. [11]. Stress point integration removes the instability due to rank deficiency which is obtained in a pure nodal integration but retains the meshfree character of the method.

\section{COUPLING PROCEDURES OF MESHFREE PARTICLE METHODS AND FEM}

\subsection{Master-slave coupling approaches}

5.1.1. Coupling by fixing particles to the FE nodes Johnson et al. [24] fixed the particles to the $\mathrm{FE}$ nodes by a simple procedure. In their method, the forces on the FE nodes $\mathbf{F}_{K}$ and the particles $\mathbf{F}_{P}$ as well as their masses and the calculation of the common accelerations are combined. The forces which act on the nodes and particles are given by

$$
\mathbf{F}_{K}=m_{K} \mathbf{a}_{K}, \mathbf{F}_{P}=m_{P} \mathbf{a}_{P}
$$

and are determined by the (already) calculated accelerations. The common acceleration of the node and corresponding particle is

$$
\mathbf{a}_{K, \text { coupling }}=\mathbf{a}_{P, \text { coupling }}=\frac{\mathbf{F}_{K}+\mathbf{F}_{P}}{m_{K}+m_{P}}
$$


One major drawback is that every particle needs a corresponding node and vice versa, so that the coupling is not valid for an arbitrary particle arrangement.

5.1.2. Master slave coupling We consider here another possibility to fix the particles rigidly to the elements so that an arbitrary nodal arrangement is possible. We follow a master slave procedure as explained in Belytschko et al. [10]. In this coupling method the particles adjacent to the FEM model are rigidly connected to the FEM interface (see figure 1). Therefore the particles are considered as slave nodes while the FEM nodes are the master nodes. As proposed in [10] the slave nodes are updated after master nodes have been updated in an explicit method.

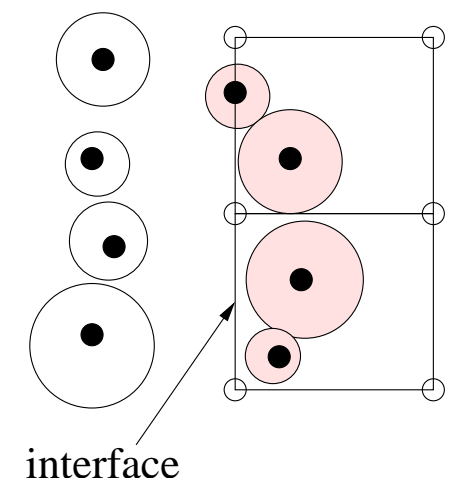

Figure 1. 2D illustration of particle/FEM interaction, gray particles are slave

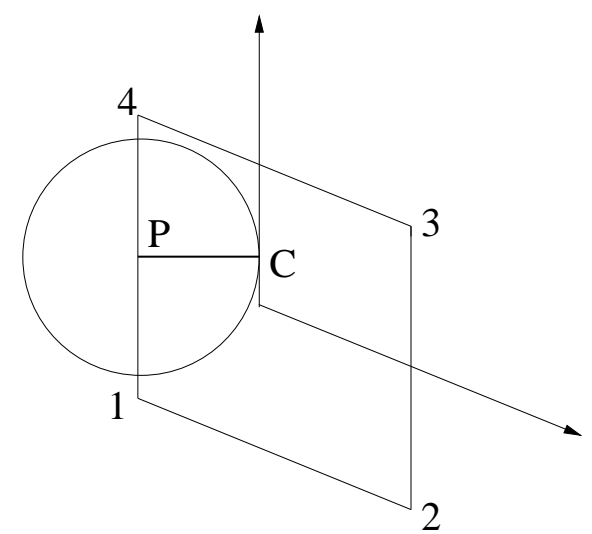

Figure 2. Projection of particle center $\mathrm{P}$ onto interface to finite elements

In the initialization, the coordinates of the closest point projection of the initial position of each particle $P$ on the finite element/meshfree interface has to be obtained as illustrated in figure 2. For convenience, a generic surface with element coordinates $\left(\xi_{c}, \eta_{C}\right)$ on the surface is considered, assumed that $\xi= \pm 1$. In the first step, the element coordinates of the projection of 
particle $P$ onto the element surface, denoted by $C$, must be found (see figure 2 ), it corresponds to the closest point projection of $P$ onto $C$. The location of this point at any time can be found by:

$$
\mathbf{x}=\sum_{J=1}^{N} N_{I}\left(\xi_{C}, \eta_{C}, \pm 1\right) \mathbf{x}_{I}
$$

The velocities of the point $C$ are given at any time by

$$
\mathbf{v}=\sum_{J=1}^{N} N_{I}\left(\xi_{C}, \eta_{C}, \pm 1\right) \mathbf{v}_{I}
$$

To obtain the velocities of the particles the master-slave algorithm (see [10]) is applied. Since $P$ is a slave node, its velocities at any time can be expressed in terms of the velocities of the nodes $I$ of the finite element $(I=1 . .4)$. The relationship will be done in two steps. First $\mathbf{v}_{P}^{S}$ is expressed in terms of $\mathbf{v}_{C}^{S}$, then $\mathbf{v}_{C}^{S}$ in terms of the master nodes $\mathbf{v}_{I}^{M}$ where the superscripts $S$ and $M$ denote slave and master nodes, respectively. Since PC is a line in a rigid body, the velocity of the slave particle are given by:

$$
\mathbf{v}_{P}^{S}=\mathbf{v}_{C}^{S}+\omega\left(\mathbf{x}_{P}-\mathbf{x}_{C}\right), \omega_{P}=\omega_{C}
$$

when $\omega$ denotes the angular velocity of the line PC. The above can be written in matrix form as:

$$
\left[\begin{array}{c}
\mathbf{v}_{P}^{S} \\
\omega_{P}^{S}
\end{array}\right]=\left[\begin{array}{cc}
\mathbf{I} & \boldsymbol{\Omega}_{P C} \\
0 & \mathbf{I}
\end{array}\right]\left[\begin{array}{l}
\mathbf{v}_{C} \\
\omega_{C}
\end{array}\right]
$$

We define

$$
\mathbf{T}_{1}=\left[\begin{array}{cc}
\mathbf{I} & \boldsymbol{\Omega}_{P C} \\
0 & \mathbf{I}
\end{array}\right]
$$

where

$$
\boldsymbol{\Omega}_{P C}=\left[\begin{array}{ccc}
0 & z_{P C} & y_{P C} \\
-z_{P C} & 0 & x_{P C} \\
-y_{P C} & -x_{P C} & 0
\end{array}\right]
$$

The velocity of point $C$ can be expressed in terms of the shape functions of the surface nodes:

$$
\mathbf{v}_{C}=\sum_{J=1}^{N} N_{I}(\xi, \eta, 1) \mathbf{v}_{I}^{M} \quad, \quad \omega_{C}=\sum_{J=1}^{N} \mathbf{A}_{I} \mathbf{v}_{I}^{M}
$$

where A will be defined later, see eq. (29) and eq. (30).

Combining these, we have:

$$
\left[\begin{array}{c}
\mathbf{v}_{C} \\
\omega_{C}
\end{array}\right]=\left[\begin{array}{c}
\mathbf{N}_{I} \mathbf{I} \\
\mathbf{A}_{I}
\end{array}\right]\left[\mathbf{v}_{I}^{M}\right]
$$

where 


$$
\mathbf{T}_{2}=\left[\begin{array}{c}
\mathbf{N}_{I} \mathbf{I} \\
\mathbf{A}_{I}
\end{array}\right]
$$

Considering the particle velocities at point $P$ and $C$ we obtain:

$$
\left[\begin{array}{c}
\mathbf{v}_{P}^{S} \\
\omega_{P}^{S}
\end{array}\right]=\sum_{J=1}^{N} \mathbf{T}_{1} \mathbf{T}_{2} \mathbf{v}_{I}^{M}
$$

where

$$
\mathbf{T}_{I}=\mathbf{T}_{1}\left[\begin{array}{c}
N_{I}\left(\xi_{C}, \eta_{C}, \pm 1\right) \\
\mathbf{A}_{I}\left(\xi_{C}, \eta_{C}, \pm 1\right)
\end{array}\right]
$$

From work conjugacy, it follows that any nodal forces on particle $P$ should be transformed and added to node $I$, so

$$
\mathbf{f}_{I}^{M}=\sum_{J \in S} \mathbf{T}_{J}^{T}\left[\begin{array}{c}
\mathbf{f}_{P}^{S} \\
\mathbf{m}_{P}^{S}
\end{array}\right] \forall J \in S
$$

where $J$ are the supporting nodes in the domain $S$. The angular velocity can be calculated by dividing the quadrilateral surface into two triangles as shown in figure 3 . The normal $\mathbf{n}$ of the triangle $\overline{123}$ is given then by:

$$
\mathbf{n}=\frac{\mathbf{x}_{12} \times \mathbf{x}_{31}}{\left\|\mathbf{x}_{12} \times \mathbf{x}_{31}\right\|}
$$

so that the angular velocity is

$$
\omega=\dot{\mathbf{n}}
$$

with

$$
\dot{\mathbf{n}}=\left(\mathbf{n}^{t+\Delta t}-\mathbf{n}^{t}\right) / \triangle t
$$

\subsection{Compatibility coupling (Coupling via ramp functions)}

Consider a domain of problem with a hybrid discretization between finite elements and particles as illustrated in figure 4 . The superscripts $F E$ and $P$ indicate the domain for the finite elements and particles, respectively. The transition region is designates by a $\Omega^{B}, \Omega^{P}$ denotes the particle domain and $\Omega^{F E}$ is the element domain. The element and particle boundary is $\Gamma^{F E}$ and $\Gamma^{P}$, respectively. The weak form of the momentum equation is the standard principle of virtual work: find $\mathbf{u} \in \mathcal{V}$ such that

$$
\delta W=\delta W_{i n t}-\delta W_{e x t}+\delta W_{k i n}=0 \quad \forall \delta \mathbf{u} \in \mathcal{V}_{0}
$$

where

$$
\begin{aligned}
\mathcal{V}= & \left\{\mathbf{u}(\cdot, t) \mid \mathbf{u}(\cdot, t) \in \mathcal{H}^{1}, \mathbf{u}(\cdot, t)=\overline{\mathbf{u}}(t) \text { on } \Gamma_{0}^{u}\right\} \\
\mathcal{V}_{0}= & \left\{\delta \mathbf{u} \mid \delta \mathbf{u} \in \mathcal{V}, \delta \mathbf{u}=0 \text { on } \Gamma_{u}\right\} \\
& \delta W_{\text {int }}=\int_{\Omega_{0}}\left(\nabla_{0} \otimes \delta \mathbf{u}\right)^{T}: \mathbf{P} d \Omega_{0}
\end{aligned}
$$




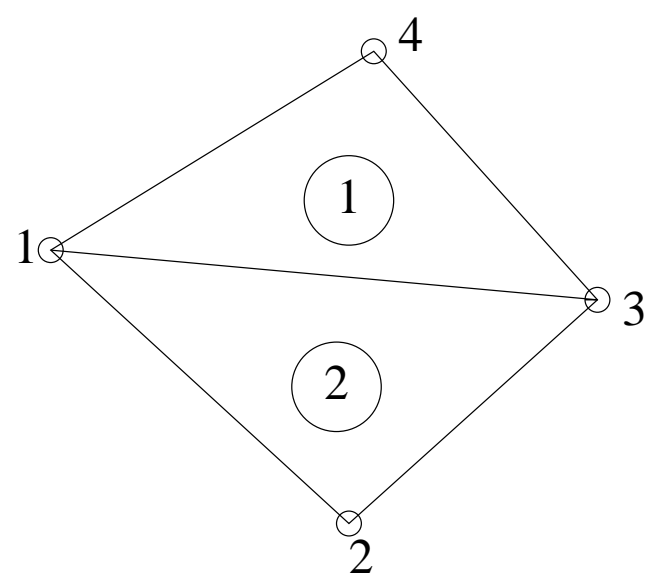

Figure 3. Computation of the angular velocity in terms of the normal

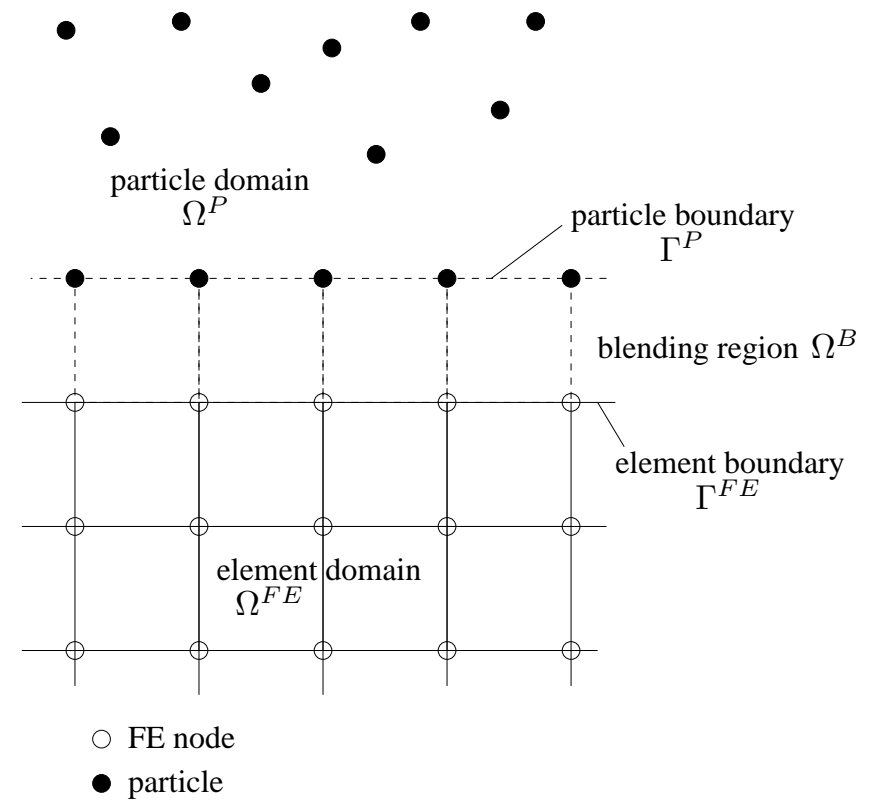

Figure 4. Coupling of Finite Elements and Particles via ramp functions

$$
\begin{gathered}
\delta W_{\text {ext }}=\int_{\Omega_{0}} \varrho_{0} \mathbf{b} \cdot \delta \mathbf{u} d \Omega_{0}+\int_{\Gamma_{0}^{t}} \overline{\mathbf{t}}_{0} \cdot \delta \mathbf{u} d \Gamma_{0} \\
\delta W_{k i n}=\int_{\Omega_{0}} \varrho_{0} \delta \mathbf{u} \cdot \ddot{\mathbf{u}} d \Omega_{0}
\end{gathered}
$$


where the prefix $\delta$ denotes the test function and $W_{\text {ext }}$ is the external energy, $W_{\text {int }}$ the internal energy and $W_{k i n}$ the kinetic energy. The approximation of the trial functions are given in section 3. The test functions have a similar structure. In the interface region, an approximation is given by:

$$
\mathbf{u}^{h}=\mathbf{u}^{F E}(\mathbf{X})+R(\mathbf{X})\left(\mathbf{u}^{P}(\mathbf{X})-\mathbf{u}^{F E}(\mathbf{X})\right) \quad, \quad \mathbf{X} \in \Omega_{B}
$$

where $\mathbf{u}^{F E}$ and $\mathbf{u}^{P}$ are the finite element and particle approximations for $\mathbf{u}$ in the transition region and $R(\mathbf{X})$ is a ramp function, so that $R(\mathbf{X})=1, \mathbf{X} \in \Gamma_{P}$ and $R(\mathbf{X})=0, \mathbf{X} \in \Gamma_{F E}$. It is constructed with the use of a linear ramp function along the interface element boundaries so that continuity is ensured:

$$
R(\mathbf{X})=3 r^{2}(\mathbf{X})-2 r^{3}(\mathbf{X})
$$

with

$$
r(\mathbf{X})=\sum_{J \in S_{\Gamma} P} N_{J}(\mathbf{X})
$$

where $S_{\Gamma^{P}}$ is the set of nodes on $\Gamma^{P}$. Substituting the FE approximations and the meshfree approximation into eq. (39) the approximation in the transition region is obtained:

$$
\mathbf{u}^{h}(\mathbf{X})=\sum_{J} \tilde{N}_{I}(\mathbf{X}) \mathbf{u}_{I}, \mathbf{X}_{I} \in \Omega^{B}
$$

with the interface shape function

$$
\begin{gathered}
\tilde{N}_{I}(\mathbf{X})=(1-R(\mathbf{X})) N_{I}(\xi(\mathbf{X}))+R(\mathbf{X}) N_{I}(\mathbf{X}) \quad \mathbf{X} \in \Omega^{B} \\
\tilde{N}_{I}(\mathbf{X})=R(\mathbf{X}) N_{I}(\mathbf{X}) \quad \mathbf{X} \notin \Omega^{B}
\end{gathered}
$$

In [4] it is proven that linear completeness is preserved in the whole domain. If the integrals for the particles are evaluated by a nodal integration with stress points, the shape functions in the blending domain have only to be evaluated at the particle boundary $\Gamma^{P}$ and element boundary $\Gamma^{F}$ and are reduced to:

$$
\begin{gathered}
\tilde{N}_{I}(\mathbf{X})=N_{I}(\mathbf{X}) \quad \mathbf{X} \in \Omega^{B} \text { on } \Gamma^{F E} \\
\tilde{N}_{I}(\mathbf{X})=0 \quad \mathbf{X} \notin \Omega^{B} \text { on } \Gamma^{F E} \\
\tilde{N}_{I}(\mathbf{X})=N(\mathbf{X}) \quad \mathbf{X} \notin \Omega^{B} \text { on } \Gamma^{P}
\end{gathered}
$$

since $R(\mathbf{X})=1$ on $\Gamma^{P}$ and $R(\mathbf{X})=0$ on $\Gamma^{F E}$. The approximation of the test functions in the blending region have the same structure. They are inserted in the weak form of the linear momentum equation. 


\subsection{Bridging domain coupling method}

5.3.1. coupling model We denote the complete domain in the initial configuration by $\Omega_{0}$ and its boundaries by $\Gamma_{0} ; \Gamma_{0}$ consists of traction boundaries $\Gamma_{0}^{t}$ and the displacement boundaries $\Gamma_{0}^{u}$. The domains are subdivided into the subdomains treated by finite element methods, $\Omega_{0}^{F E}$, and that treated by particle methods, $\Omega_{0}^{P}$; the latter is the domain encompassed by the particles of the model. The intersection of these two domains is denoted by $\Omega_{0}^{i n t}$ in the initial configuration, $\Omega^{i n t}$ in the current configuration; $\Omega^{i n t}$ is often called the overlapping subdomain (or bridging domain), see Belytschko and Xiao [6]; $\Gamma_{0}^{\alpha}$ denotes the edge of the finite element domain; an example of a model is shown in figure 5.

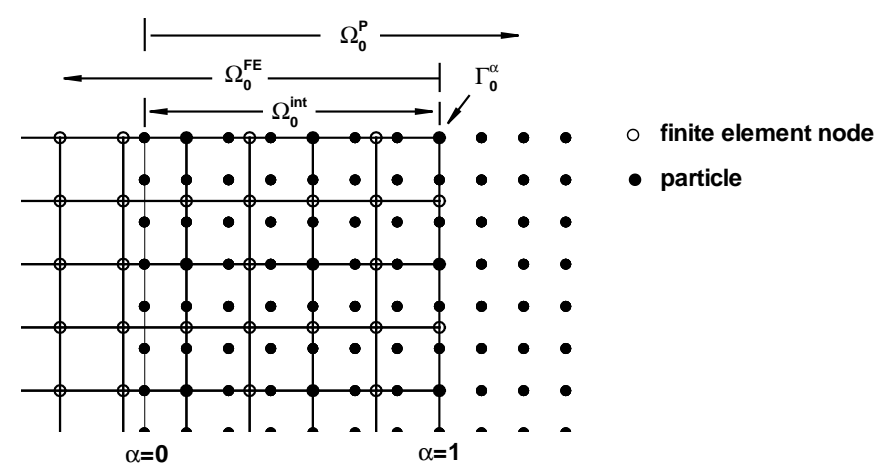

Figure 5. Finite element model coupled with particle method

In expressing the total internal potential energy of the system we employ a scaling parameter $\alpha$ in the overlapping subdomain. The parameter $\alpha$ is defined as $\alpha=\frac{l(\mathbf{X})}{l_{0}}$ where $l(\mathbf{X})$ is the least square of projection of $\mathbf{X}$ onto $\Gamma_{0}^{\alpha}$ as shown in figure 5 . The scaling parameter $\alpha$ is unity at the edge of the finite element domain and vanishes at the other edge of $\Omega_{0}^{\text {int }}$; it is important that $\Omega_{0}^{\text {int }}$ includes the last line of particles.

In the absence of heat transmission the conservation of energy of governing equations in the entire domain is:

$$
W^{i n t}=\int_{\Omega_{0}^{F E}} \beta^{F E} \mathbf{F}^{T} \cdot \mathbf{P} d \Omega_{0}^{F E}+\int_{\Omega_{0}^{P}} \beta^{P} \mathbf{F}^{T} \cdot \mathbf{P} d \Omega_{0}^{P}
$$

where the scaling multiplier field $\beta$ is defined as

$$
\begin{aligned}
\beta^{F E}(\mathbf{X}) & =\left\{\begin{array}{lll}
0 & \text { in } & \Omega_{0}^{P} \\
1-\alpha & \text { in } & \Omega_{0}^{i n t} \\
1 & \text { in } & \Omega_{0}^{F E}-\Omega_{0}^{i n t}
\end{array}\right. \\
\beta^{P}(\mathbf{X}) & =\left\{\begin{array}{lll}
0 & \text { in } & \Omega_{0}^{F E} \\
\alpha & \text { in } & \Omega_{0}^{i n t} \\
1 & \text { in } & \Omega_{0}^{P}-\Omega_{0}^{i n t}
\end{array}\right.
\end{aligned}
$$


The external energy is:

$$
\begin{aligned}
W^{e x t} & =\int_{\Omega_{0}^{F E}} \beta^{F E} \rho_{0} \mathbf{b} \cdot \mathbf{u} d \Omega_{0}^{F E}+\int_{\Omega_{0}^{P}} \beta^{P} \rho_{0} \mathbf{b} \cdot \mathbf{u} d \Omega_{0}^{P} \\
& +\int_{\Gamma_{0}^{F E}} \beta^{F E} \overline{\mathbf{t}} \cdot \mathbf{u} d \Gamma_{0}^{F E}+\int_{\Gamma_{0}^{P}} \beta^{P} \overline{\mathbf{t}} \cdot \mathbf{u} d \Gamma_{0}^{P}
\end{aligned}
$$

In $\Omega_{0}^{\text {int }}$ the displacements can be approximated in the terms of shape functions $N_{I}(\mathbf{X})$ of finite element method or kernel functions $w_{I}(\mathbf{X})$ of particle methods respectively by:

$$
\begin{aligned}
\mathbf{u}^{F E}(\mathbf{X}, t) & =\sum_{I} N_{I}(\mathbf{X}) \mathbf{u}_{I}^{F E}(t) \\
\mathbf{u}^{P}(\mathbf{X}, t) & =\sum_{I} w_{I}(\mathbf{X}) \mathbf{u}_{I}^{P}(t)
\end{aligned}
$$

Therefore, the constraints condition in $\Omega_{0}^{i n t}$ at the discrete position of particles are

$$
\mathbf{g}_{I}=\left\{g_{i I}\right\}=\left\{u_{i I}^{F E}-u_{i I}^{P}\right\}=\left\{\sum_{J} N_{J I} u_{i J}^{F E}-\sum_{K} w_{K I} u_{i K}^{P}\right\}
$$

The Lagrange multiplier field is also expressed in terms of shape functions denoted by $\Lambda_{I}(\mathbf{X})$ :

$$
\lambda_{i}(\mathbf{X}, t)=\sum_{I} \Lambda_{I}(\mathbf{X}) \bar{\lambda}_{i I}(t)
$$

Generally, the shape functions for the Lagrange multiplier field $\Lambda_{I}(\mathbf{X})$ will differ from that for the displacement, $N_{I}(\mathbf{X})$ or $w_{I}(\mathbf{X})$, and they must satisfy the LBB conditions. The Lagrange multiplier field is usually represented by inserting finite elements in the intersection domain and the finite element approximation is applied on the particles of the overlapping subdomain. To distinguish the Lagrange multiplier field $\lambda_{i}$ in eq. (53), $\bar{\lambda}_{i I}$ is denoted as the unknown Lagrange multiplier at the Lagrange multiplier nodes.

\subsubsection{Discrete Equations The energy function for augmented Lagrangian method is}

$$
W_{A L}=W^{i n t}-W^{e x t}+\lambda^{T} \mathbf{g}+\frac{1}{2} p \mathbf{g}^{T} \mathbf{g}
$$

where $p$ is the penalty parameter. If $p=0$, eq. (54) will be identical to the expression of energy function for Lagrange multiplier method.

The discrete equations are then obtained by setting the derivatives of $W_{A L}$ with respect to 
$\mathbf{u}_{\mathbf{I}}$ and $\lambda_{I}$ to zero. This gives

$$
\begin{aligned}
\frac{\partial W_{A L}}{\partial u_{i I}^{F E}} & =\left(F_{i I}^{i n t}-F_{i I}^{e x t}\right)+\sum_{L}\left[\left(\sum_{K} \Lambda_{K L} \bar{\lambda}_{K}\right) N_{I L}\right] \\
& +p \sum_{L}\left[\left(\sum_{K} N_{K L} u_{i K}^{F E}-\sum_{K} w_{K L} u_{i K}^{P}\right) N_{I L}\right]=0 \\
\frac{\partial W_{A L}}{\partial u_{i I}^{P}} & =\left(f_{i I}^{i n t}-f_{i I}^{e x t}\right)-\sum_{L}\left[\left(\sum_{K} \Lambda_{K L} \bar{\lambda}_{K}\right) w_{I L}\right] \\
& -p \sum_{L}\left[\left(\sum_{K} N_{K L} u_{i K}^{F E}-\sum_{K} w_{K L} u_{i K}^{P}\right) w_{I L}\right]=0 \\
\frac{\partial W_{A L}}{\partial \bar{\lambda}_{i I}} & =\sum_{L} \Lambda_{I L}\left[\sum_{K} N_{K L} u_{i K}^{F E}-\sum_{K} w_{K L} u_{i K}^{P}\right]=0
\end{aligned}
$$

where

$$
N_{K I}=N_{K}\left(\mathbf{X}_{I}\right) \quad \Lambda_{K I}=\Lambda_{K}\left(\mathbf{X}_{I}\right)
$$

$\mathbf{F}^{i n t}$ and $\mathbf{F}^{e x t}$ are internal and external force in finite element subdomain $\Omega_{0}^{F E}$ and they are expressed as:

$$
\begin{aligned}
\mathbf{F}_{i I}^{i n t} & =\int_{\Omega_{0}^{F E}} \beta^{F E} N_{I, j}(\mathbf{X}) \mathbf{P}_{j i}(\mathbf{X}) d \Omega_{0}^{F E} \\
\mathbf{F}_{i I}^{e x t} & =\int_{\Omega_{0}^{F E}} \beta^{F E} \rho_{0} b_{i} d \Omega_{0}^{F E}+\int_{\Gamma_{0}^{t}} \beta^{F E} \bar{t}_{i} d \Gamma_{0}^{t}
\end{aligned}
$$

and $\mathbf{f}^{\text {int }}$ and $\mathbf{f}^{\text {ext }}$ are internal and external force in particle subdomain $\Omega_{0}^{P}$ and they are expressed as:

$$
\begin{aligned}
\mathbf{f}_{i I}^{i n t} & =\int_{\Omega_{0}^{P}} \beta^{P} w_{I, j}(\mathbf{X}) \mathbf{P}_{j i}(\mathbf{X}) d \Omega_{0}^{P} \\
\mathbf{f}_{i I}^{e x t} & =\int_{\Omega_{0}^{P}} \beta^{P} \rho_{0} b_{i} d \Omega_{0}^{P}+\int_{\Gamma_{0}^{t}} \beta^{P} \bar{t}_{i} d \Gamma_{0}^{t}
\end{aligned}
$$

Letting $\mathbf{d}$ denote the array of $\mathbf{u}$, the increments in the internal nodal force can be approximated in terms of increments in the nodal displacement by stiffness matrices:

$$
\begin{aligned}
\Delta \mathbf{F}_{I}^{i n t} & =\sum_{J} \mathbf{K}_{I J}^{F E} \Delta \mathbf{u}_{J}^{F E} \quad \text { or } \quad \Delta \mathbf{F}^{i n t}=\mathbf{K}^{F E} \Delta \mathbf{d}^{F E} \\
\Delta \mathbf{f}_{I}^{i n t} & =\sum_{J} \mathbf{K}_{I J}^{P} \Delta \mathbf{u}_{J}^{P} \quad \text { or } \quad \Delta \mathbf{f}^{i n t}=\mathbf{K}^{P} \Delta \mathbf{d}^{P}
\end{aligned}
$$


where $\mathbf{K}^{F E}$ and $\mathbf{K}^{P}$ are tangent stiffness matrices given by

$$
\begin{gathered}
\mathbf{K}^{F E}=\left[\begin{array}{llll}
\mathbf{K}_{11}^{F E} & \mathbf{K}_{12}^{F E} & & \\
\mathbf{K}_{21}^{F E} & \mathbf{K}_{22}^{F E} & & \\
& & \cdots & \\
& & & \mathbf{K}_{n n}^{F E}
\end{array}\right] \quad \mathbf{K}_{I J}^{F E}=\frac{\partial \mathbf{F}_{I}^{i n t}}{\partial \mathbf{u}_{J}^{F E}} \\
\mathbf{K}^{P}=\left[\begin{array}{llll}
\mathbf{K}_{11}^{P} & \mathbf{K}_{12}^{P} & & \\
\mathbf{K}_{21}^{P} & \mathbf{K}_{22}^{P} & & \\
& & \cdots & \\
& & \mathbf{K}_{m m}^{P}
\end{array}\right] \quad \mathbf{K}_{I J}^{P}=\frac{\partial \mathbf{f}_{I}^{i n t}}{\partial \mathbf{u}_{J}^{P}} \\
\mathbf{d}^{F E}=\left\{\begin{array}{l}
\mathbf{d}_{1}^{F E} \\
\mathbf{d}_{2}^{F E} \\
\cdots \\
\mathbf{d}_{n}^{F E}
\end{array}\right\} \quad \mathbf{d}_{I}^{F E}=\left\{\begin{array}{l}
u_{x I}^{F E} \\
u_{y I}^{F E}
\end{array}\right\} \quad \mathbf{d}^{P}=\left\{\begin{array}{c}
\mathbf{d}_{1}^{P} \\
\mathbf{d}_{2}^{P} \\
\cdots \\
\mathbf{d}_{m}^{P}
\end{array}\right\} \quad \mathbf{d}_{I}^{P}=\left\{\begin{array}{l}
u_{x I}^{P} \\
u_{y I}^{P}
\end{array}\right\}
\end{gathered}
$$

The system can then be written as

$$
\left[\begin{array}{ccc}
\mathbf{A}_{11} & \mathbf{A}_{12} & \mathbf{L}^{F E^{T}} \\
\mathbf{A}_{21} & \mathbf{A}_{22} & \mathbf{L}^{P^{T}} \\
\mathbf{L}^{F E} & \mathbf{L}^{P} & 0
\end{array}\right]\left\{\begin{array}{c}
\Delta \mathbf{d}^{F E} \\
\Delta \mathbf{d}^{P} \\
\Delta \bar{\lambda}
\end{array}\right\}=\left\{\begin{array}{c}
-\mathbf{r}^{F E} \\
-\mathbf{r}^{P} \\
-\mathbf{g}
\end{array}\right\}
$$

If we let $d_{i}$ denote $u_{k P}$ and $d_{j}$ denote $u_{l Q}$, the ingredients of eq. (68) can be expressed as:

$$
\begin{aligned}
& \mathbf{r}^{F E}=\mathbf{F}^{i n t}-\mathbf{F}^{e x t}+\lambda^{T} \mathbf{G}^{F E}+p \mathbf{g}^{T} \mathbf{G}^{F E} \\
& \mathbf{r}^{P}=\mathbf{f}^{\text {int }}-\mathbf{f}^{e x t}+\lambda^{T} \mathbf{G}^{P}+p \mathbf{g}^{T} \mathbf{G}^{P} \\
& \mathbf{g}=\left\{\bar{g}_{i I}\right\}=\left\{\sum_{K} \Lambda_{I K} g_{i K}\right\} \\
& \mathbf{A}_{11}= \mathbf{K}^{F E}+p \mathbf{G}^{F E^{T}} \mathbf{G}^{F E} \\
& \mathbf{A}_{12}=p \mathbf{G}^{F E^{T}} \mathbf{G}^{P} \\
& \mathbf{A}_{21}=p \mathbf{G}^{P^{T}} \mathbf{G}^{F E} \\
& \mathbf{A}_{22}=\mathbf{K}^{P}+p \mathbf{G}^{P^{T}} \mathbf{G}^{P} \\
& \lambda_{i I}=\sum_{K} \Lambda_{K}\left(\mathbf{X}_{I}\right) \bar{\lambda}_{i K} \\
& \mathbf{K}^{F E}=\left[\frac{\partial \mathbf{F}^{\mathbf{i n t}}}{\partial \mathbf{d}^{F E}}\right]=\left[\frac{\partial F_{i I}^{i n t}}{\partial u_{l Q}^{F E}}\right]=\left[\int_{\Omega_{0}^{F E}} \beta^{F E} N_{I, j} \bar{C}_{j i l k} N_{Q, k} d \Omega_{0}^{F E}\right] \\
& \mathbf{K}^{P}=\left[\frac{\partial \mathbf{f}^{\mathbf{i n t}}}{\partial \mathbf{d}^{P}}\right]=\left[\frac{\partial f_{i I}^{i n t}}{\partial u_{l Q}^{P}}\right]=\left[\int_{\Omega_{0}^{P}} \beta^{P} w_{I, j} \bar{C}_{j i l k} w_{Q, k} d \Omega_{0}^{P}\right] \\
& \mathbf{L}^{F E}=\left[\sum_{L} \Lambda_{I L} \frac{\partial \mathbf{g}_{L}}{\partial d_{i}^{F E}}\right]=\left[\sum_{L} \Lambda_{I L} \frac{\partial g_{j L}}{\partial d_{i}^{F E}}\right]=\left[\sum_{L} \Lambda_{I L} \frac{\partial g_{L}}{\partial u_{k P}^{F E}}\right]=\left[\sum_{L} \Lambda_{I L} N_{P I} \delta_{j k}\right](79) \\
& \mathbf{L}^{P}=\left[\sum_{L} \Lambda_{I L} \frac{\partial \mathbf{g}_{L}}{\partial d_{i}^{P}}\right]=\left[\sum_{L} \Lambda_{I L} \frac{\partial g_{j L}}{\partial d_{i}^{P}}\right]=\left[\sum_{L} \Lambda_{I L} \frac{\partial g_{L}}{\partial u_{k P}^{P}}\right]=\left[-\sum_{L} \Lambda_{I L} w_{P I} \delta_{j k}\right]
\end{aligned}
$$




$$
\begin{aligned}
\mathbf{G}^{F E} & =\left[\frac{\partial \mathbf{g}_{I}}{\partial d_{i}^{F E}}\right]=\left[\frac{\partial g_{j I}}{\partial u_{k P}^{F E}}\right]=\left[N_{P I} \delta_{j k}\right] \\
\mathbf{G}^{P} & =\left[\frac{\partial \mathbf{g}_{I}}{\partial d_{i}^{P}}\right]=\left[\frac{\partial g_{j I}}{\partial u_{k P}^{P}}\right]=\left[-w_{P I} \delta_{j k}\right]
\end{aligned}
$$

5.3.3. Explicit time integration In dynamics, the Lagrangian of the system is

$$
L=W^{k i n}-W^{i n t}+W^{e x t}
$$

where $W^{k i n}=\sum_{I} m_{I} \frac{1}{2} \dot{d}_{I}^{2}$ is kinetic energy.

Therefore, the Lagrangian for the coupling model is

$$
L=L^{F E}+L^{P}=\beta^{F E} W_{F E}^{k i n}-\beta^{F E} W_{F E}^{i n t}+\beta^{F E} W_{F E}^{e x t}+\beta^{P} W_{P}^{k i n}-\beta^{P} W_{P}^{i n t}+\beta^{P} W_{P}^{e x t}
$$

The Lagrangian for augmented lagrangian method is

$$
L^{A L}=L+\lambda^{T} \mathbf{g}+\frac{1}{2} p \mathbf{g}^{T} \mathbf{g}
$$

In Lagrangian mechanics the equations of motion are written as

$$
\frac{d}{d t}\left(\frac{\partial L}{\partial \dot{d}}\right)=\frac{\partial L}{\partial d}
$$

Then, we obtain the equations of motion by substituting eq. (84) into eq. (85):

$$
\begin{gathered}
\overline{\mathbf{M}} \ddot{\mathbf{u}}^{F E}+\mathbf{F}^{i n t}-\mathbf{F}^{e x t}-\mathbf{F}^{C}=0 \\
\overline{\mathbf{m}} \ddot{\mathbf{u}}^{P}+\mathbf{f}^{i n t}-\mathbf{f}^{e x t}-\mathbf{f}^{C}=0
\end{gathered}
$$

where

$$
\begin{aligned}
\bar{M}_{I J} & =\int_{\Omega_{0}^{F E}} \beta^{F E} \rho_{0} N_{I}(X) N_{J}(X) d \Omega_{0}^{F E} \\
\bar{m}_{I J} & =\int_{\Omega_{0}^{P}} \beta^{P} \rho_{0} w_{I}(X) w_{J}(X) d \Omega_{0}^{P}
\end{aligned}
$$

the internal and external forces are define as before. $\mathbf{F}^{C}$ and $\mathbf{f}^{C}$ are extra forces from the constraints

$$
\begin{aligned}
\mathbf{F}^{C} & =\lambda^{T} \frac{\partial \mathbf{g}}{\partial \mathbf{u}^{\mathbf{F E}}}+p \mathbf{g}^{T} \frac{\partial \mathbf{g}}{\partial \mathbf{u}^{\mathbf{F E}}}=\lambda^{T} \mathbf{G}^{F E}+p \mathbf{g}^{T} \mathbf{G}^{F E} \\
\mathbf{f}^{C} & =\lambda^{T} \frac{\partial \mathbf{g}}{\partial \mathbf{u}^{\mathbf{P}}}+p \mathbf{g}^{T} \frac{\partial \mathbf{g}}{\partial \mathbf{u}^{\mathbf{P}}}=\lambda^{T} \mathbf{G}^{P}+p \mathbf{g}^{T} \mathbf{G}^{P}
\end{aligned}
$$

Equation 87 and equation 86 can be written in a general form:

$$
\mathbf{M} \ddot{\mathbf{d}}=\mathbf{f}^{e x t}-\mathbf{f}^{i n t}+\mathbf{f}^{C}
$$

In time step $n+1$, we first obtain the trial velocities by

$$
\overline{\mathbf{v}}=\mathbf{v}_{(n)}+\mathbf{M}^{-1} \Delta t\left(\mathbf{f}^{e x t}-\mathbf{f}^{i n t}+p \mathbf{g}^{T} \mathbf{G}\right)
$$


The trial velocities need to be corrected by applying the constraint equations, which are written in time derivative form

$$
\dot{g}_{I}=\mathbf{v}_{I}^{F E}-\mathbf{v}_{I}^{P}=\sum_{J} N_{J I} \mathbf{v}_{J}^{F E}-\sum_{J} w_{J I} \mathbf{v}_{J}^{P}=0
$$

where the velocities are the values at time step $n+1$ and given by:

$$
\begin{aligned}
\mathbf{v}_{(n+1)} & =\mathbf{v}_{(n)}+\mathbf{M}^{-1} \Delta t\left(\mathbf{f}^{e x t}-\mathbf{f}^{i n t}+\lambda^{T} \mathbf{G}+p \mathbf{g}^{T} \mathbf{G}\right) \\
& =\overline{\mathbf{v}}+\mathbf{M}^{-1} \Delta t \lambda^{T} \mathbf{G}
\end{aligned}
$$

Substituting equation 95 into equation 94, the Lagrange multiplier can be obtained by solving the following equations:

$$
\begin{gathered}
\left(\sum_{J} \frac{\Delta t}{M_{J}} w_{J I} \mathbf{G}_{L J}^{P}-\sum_{K} \frac{\Delta t}{M_{K}} N_{K I} \mathbf{G}_{L K}^{F E}\right) \lambda_{L}=\left(\sum_{J} N_{J I} \overline{\mathbf{v}}_{J}^{F E}-\sum_{K} w_{K I} \overline{\mathbf{v}}_{K}^{P}\right) \\
\lambda_{\mathbf{L}}=\left\{\begin{array}{c}
\lambda_{x L} \\
\lambda_{y L}
\end{array}\right\} \quad \overline{\mathbf{v}}_{J}^{F E}=\left\{\begin{array}{c}
\bar{v}_{x J}^{F E} \\
\bar{v}_{y J}^{F E}
\end{array}\right\} \quad \overline{\mathbf{v}}_{K}^{P}=\left\{\begin{array}{c}
\bar{v}_{x K}^{P} \\
\bar{v}_{y K}^{P}
\end{array}\right\}
\end{gathered}
$$

The Lagrange multipliers, which are set on the positions of discrete atoms, are treated as unknown variables in the dynamic algorithm. The algorithm is given in Table I.

1. initial conditions and initialization: $t=0$ and compute $\mathbf{M}$;

2. get $\mathbf{f}^{\mathbf{i n t}}$, $\mathbf{f}^{\mathbf{e x t}}$ and $\mathbf{f}^{\mathbf{C}}$ which is from equation 92 ;

3. compute trial velocities from eq. 93;

4. compute the unknown of Lagrange multipliers $\Delta \lambda$ from eq. 96;

5. update velocities from eq. 95;

6. apply boundary conditions;

7. output; if simulation does not complete, go to 2

Table I. Explicit algorithm for dynamics with coupling method

\subsection{Compatibility coupling:Coupling with Lagrange multipliers}

A coupling approach where no ramp functions are needed was first proposed by Hegen et al. [21]. Rabczuk and Belytschko [44] used this approach to couple EFG nodes and finite elements to model the bond behavior in reinforced concrete beams in statics. In this method, relative displacements between the particles and the elements are allowed. Xiao [51] developed an explicit method based on Lagrange multipliers (similar to the approach of Hegen et al. [21]) which is a simplified version of the bridging domain coupling. He called this method edge-to-edge coupling. The main difference is that the FE and particle domain do not overlap which simplifies the method enormously. However, for wave propagation problems, the bridging domain coupling is more accurate. We will review the coupling via Lagrange multipliers as in [44] and extend it to dynamics. 


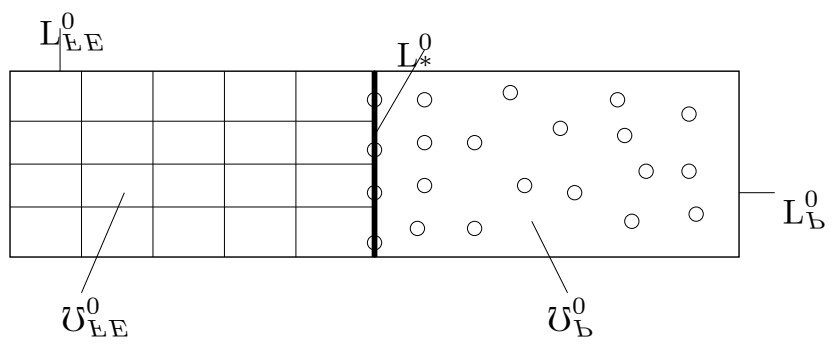

Figure 6. Coupling of particle and finite elements

For the static case, the potential to be minimized is

$$
W=W^{i n t}-W^{e x t}+\boldsymbol{\lambda}^{T} \mathbf{g}
$$

where $W^{\text {int }}$ is the internal and $W^{\text {ext }}$ is the external energy. The last term on the RHS are the constraints. In the dynamic case, an inertia term is added. The Lagrange multipliers are denoted by $\boldsymbol{\lambda}$ and $\mathbf{g}=\mathbf{u}^{F E}-\mathbf{u}^{P}$ is the gap of the particle and the finite element domain along the common boundary as illustrated in figure 6 . The Lagrange multipliers are located at the particle positions and are

$$
\mathbf{g}_{h}=\sum_{J=1}^{N} N_{J}^{F E}(\mathbf{X}, t) \mathbf{u}_{J}^{F E}-\sum_{J \in S} N_{J}^{P}(\mathbf{X}, t) \mathbf{u}_{J}^{P}
$$

The Lagrange multiplier estimates are placed at the particle position and finite element shape functions are used to discretize the Lagrange multiplier field $\delta \boldsymbol{\lambda}$ :

$$
\delta \boldsymbol{\lambda}_{h}^{P}(\mathbf{X}, t)=\sum_{J=1}^{N} N_{J}^{F E}(\mathbf{X}, t) \delta \boldsymbol{\Lambda}_{J}(t)
$$

Note that for the interpolation in eq. (100), the position of the Lagrange multipliers in the local element coordinate system has to be known. The test and trial functions are

$$
\begin{aligned}
\delta \mathbf{u}_{h}(\mathbf{X}, t) & =\sum_{J=1}^{N} N_{J}^{F E}(\mathbf{X}, t) \delta \mathbf{u}_{J}^{F E}(t)+\sum_{J \in \mathcal{S}} N_{J}^{P}(\mathbf{X}, t) \delta \mathbf{u}_{J}^{P}(t) \\
\mathbf{u}_{h}(\mathbf{X}, t) & =\sum_{J=1}^{N} N_{J}^{F E}(\mathbf{X}, t) \mathbf{u}_{J}^{F E}(t)+\sum_{J \in \mathcal{S}} N_{J}^{P}(\mathbf{X}, t) \mathbf{u}_{J}^{P}(t)
\end{aligned}
$$

and

$$
\begin{aligned}
& N^{F E}(\mathbf{X}, t)=0 \forall \mathbf{X} \in \Omega_{0}^{P} \\
& N^{P}(\mathbf{X}, t)=0 \forall \mathbf{X} \in \Omega_{0}^{F E}
\end{aligned}
$$

where $\mathcal{S}$ is the set of nodes in the particle model. 
Minimizing eq. (98) with respect to $\mathbf{u}$ and $\boldsymbol{\lambda}$ leads to the following equations:

$$
\begin{array}{r}
\frac{\partial W}{\partial \mathbf{u}}=\frac{\partial W^{i n t}}{\partial \mathbf{u}}-\frac{\partial W^{e x t}}{\partial \mathbf{u}}+\boldsymbol{\lambda} \frac{\partial \mathbf{g}}{\partial \mathbf{u}}=\mathbf{f}^{i n t}-\mathbf{f}^{e x t}+\boldsymbol{\lambda} \frac{\partial \mathbf{g}}{\partial \mathbf{u}}=0 \\
\frac{\partial W}{\partial \boldsymbol{\lambda}}=\mathbf{g}=0
\end{array}
$$

The derivatives of $W^{\text {int }}$ and $W^{\text {ext }}$ with respect to $\mathbf{u}$ are the internal and external forces, respectively:

$$
\begin{gathered}
\mathbf{f}^{i n t}=\int_{\Omega_{0}^{P} \cup \Omega_{0}^{F E}}\left(\nabla_{0} \otimes \delta \mathbf{u}\right)^{T}: \mathbf{P} d \Omega_{0} \\
\mathbf{f}^{e x t}=\int_{\Omega_{0}^{P} \cup \Omega_{0}^{F E}} \delta \mathbf{u} \cdot \mathbf{b} d \Omega_{0}+\int_{\Gamma_{0}^{P, t} \cup \Gamma_{0}^{F E, t}} \delta \mathbf{u} \cdot \overline{\mathbf{t}}_{0} d \Gamma_{0}
\end{gathered}
$$

The additional forces $\boldsymbol{\lambda} \frac{\partial \mathbf{g}}{\partial \mathbf{u}}$ are linear combinations of the Lagrange multipliers. To obtain the discrete system of nonlinear equations we will do a linearization as described e.g. in Belytschko et al. [10]. Therefore, we take a Taylor series expansion of eq. (104) neglecting any higher order terms:

$$
\begin{array}{r}
\mathbf{f}^{i n t}-\mathbf{f}^{e x t}+\lambda \frac{\partial \mathbf{g}}{\partial \mathbf{u}}+\frac{\partial \mathbf{f}^{i n t}}{\partial \mathbf{u}} \Delta \mathbf{u}-\frac{\partial \mathbf{f}^{e x t}}{\partial \mathbf{u}} \Delta \mathbf{u}+\frac{\partial \mathbf{g}}{\partial \mathbf{u}} \Delta \boldsymbol{\lambda}+\boldsymbol{\lambda} \frac{\partial^{2} \mathbf{g}}{\partial \mathbf{u} \partial \mathbf{u}} \Delta \mathbf{u}=0 \\
\mathbf{u}+\frac{\partial \mathbf{g}}{\partial \mathbf{u}} \Delta \mathbf{u}=0
\end{array}
$$

Substituting the test and trial functions, eqs. (100), (101) and (102) into (107) we finally obtain the following system of equations:

$$
\left[\begin{array}{ccc}
\mathbf{K}^{F E}+\boldsymbol{\lambda} \frac{\partial^{2} \mathbf{g}}{\partial \mathbf{u} \partial \mathbf{u}} & 0 & \left(\mathbf{K}^{F E-F E}\right)^{T} \\
0 & \mathbf{K}^{P}+\boldsymbol{\lambda} \frac{\partial^{2} \mathbf{g}}{\partial \mathbf{u} \partial \mathbf{u}} & \left(\mathbf{K}^{F E-P}\right)^{T} \\
\mathbf{K}^{F E-F E} & \left(\mathbf{K}^{F E-P}\right)^{T} & 0
\end{array}\right] \cdot\left[\begin{array}{c}
\Delta \mathbf{u}_{J}^{F E} \\
\Delta \mathbf{u}_{J}^{P} \\
\Delta \boldsymbol{\Lambda}
\end{array}\right]=\left[\begin{array}{c}
\mathbf{f}^{e x t, F E}-\mathbf{f}^{i n t, F E}-\boldsymbol{\lambda}^{T} \mathbf{K}^{F E-F E} \\
\mathbf{f}^{e x t, P}-\mathbf{f}^{i n t, P}-\boldsymbol{\lambda}^{T} \mathbf{K}^{F E-P(108)} \\
-\mathbf{g}
\end{array}\right]
$$

where $\mathbf{K}^{F E-F E}$ and $\mathbf{K}^{F E-P}$ denotes the derivatives of $\mathbf{g}$ with respect to $\mathbf{u}$ for the finite element $\left(\mathbf{u}^{F E}\right)$ and particle domain $\left(\mathbf{u}^{P}\right)$, respectively and $\mathbf{K}^{F E}$ and $\mathbf{K}^{P}$ are the derivatives of the internal and external forces with respect to $\mathbf{u}$. Since neither $\mathbf{b}$ nor the traction boundary conditions $\overline{\mathbf{t}}$ depend on $\mathbf{u}$, the derivatives on $\mathbf{f}^{\text {ext }}$ exist only for the cohesive model. Finally we can give the matrices:

$$
\begin{aligned}
\mathbf{K}^{F E-F E} & =\int_{\Gamma_{0}^{*}}\left(\mathbf{N}^{F E}\right)^{T} \cdot \mathbf{N}^{F E} d \Gamma_{0} \\
\mathbf{K}^{F E-P} & =-\int_{\Gamma_{0}^{*}}\left(\mathbf{N}^{F E}\right)^{T} \cdot \mathbf{N}^{P} d \Gamma_{0} \\
\mathbf{K}^{P} & =\int_{\Omega_{0}^{P}}\left(\mathbf{B}^{P}\right)^{T} \mathbf{C} \mathbf{B}^{P} d \Omega_{0} \\
\mathbf{K}^{F E} & =\int_{\Omega_{0}^{F E}}\left(\mathbf{B}^{F E}\right)^{T} \mathbf{C} \mathbf{B}^{F E} d \Omega_{0}
\end{aligned}
$$


and the vectors for internal and external forces

$$
\begin{aligned}
\mathbf{f}^{e x t, F E} & =\int_{\Omega_{0}^{F E}}\left(\mathbf{N}^{F E}\right)^{T} \mathbf{b} d \Omega_{0}+\int_{\Gamma_{0}^{F E, t}}\left(\mathbf{N}^{F E}\right)^{T} \overline{\mathbf{t}}_{0} d \Gamma_{0} \\
\mathbf{f}^{e x t, P} & =\int_{\Omega_{0}^{P}}\left(\mathbf{N}^{P}\right)^{T} \mathbf{b} d \Omega_{0}+\int_{\Gamma_{0}^{P, t}}\left(\mathbf{N}^{P}\right)^{T} \overline{\mathbf{t}}_{0} d \Gamma_{0} \\
\mathbf{f}^{i n t, F E} & =\int_{\Omega_{0}^{F E}}\left(\mathbf{B}^{F E}\right)^{T} \cdot \mathbf{P} d \Omega_{0} \\
\mathbf{f}^{i n t, P} & =\int_{\Omega_{0}^{P}}\left(\mathbf{B}^{P}\right)^{T} \cdot \mathbf{P} d \Omega_{0}
\end{aligned}
$$

For dynamics, let us define the problem for every domain seperately: Find $\mathbf{u}^{i} \in H^{1}\left(\Omega^{i}\right), i=$ $F E$ or $P$ and $\boldsymbol{\lambda} \in H^{0}\left(\Gamma^{*}\right)$ so that

$$
\begin{array}{r}
\int_{\Omega_{0}^{F E}} \varrho_{0}^{F E} \delta \mathbf{u}^{F E} \cdot \ddot{\mathbf{u}}^{F E} d \Omega_{0}+\int_{\Omega_{0}^{F E}} \nabla \delta \mathbf{u}^{F E}: \mathbf{P}^{F E} d \Omega_{0}-\int_{\Omega_{0}^{F E}} \delta \mathbf{u}^{F E} \cdot \mathbf{b}^{F E} d \Omega_{0}- \\
\int_{\Gamma_{0}^{1 t}} \delta \mathbf{u}^{F E} \cdot \overline{\mathbf{t}}^{F E} d \Gamma-\int_{\Gamma_{*}} \delta \boldsymbol{\lambda} \cdot \mathbf{u}^{F E} d \Gamma-\int_{\Gamma_{*}} \delta \mathbf{u}^{F E} \cdot \boldsymbol{\lambda} d \Gamma=0 \\
\int_{\Omega_{0}^{p}} \varrho_{0}^{P} \delta \mathbf{u}^{P} \cdot \ddot{\mathbf{u}}^{P} d \Omega_{0}+\int_{\Omega_{0}^{P}} \nabla \delta \mathbf{u}^{P}: \mathbf{P}^{P} d \Omega_{0}-\int_{\Omega_{0}^{P}} \delta \mathbf{u}^{P} \cdot \mathbf{b}^{P} d \Omega_{0}- \\
\int_{\Gamma_{0}^{P t}} \delta \mathbf{u}^{P} \cdot \overline{\mathbf{t}}^{P} d \Gamma+\int_{\Gamma_{*}} \delta \boldsymbol{\lambda} \cdot \mathbf{u}^{P} d \Gamma+\int_{\Gamma_{*}} \delta \mathbf{u}^{P} \cdot \boldsymbol{\lambda} d \Gamma=0
\end{array}
$$

for all $\delta \mathbf{u}^{i} \in H^{1}\left(\Omega^{i}\right), i=F E$ or $P$ and $\delta \boldsymbol{\lambda} \in H^{0}\left(\Gamma_{*}\right)$ where the superscript $i$ indicates the corresponding domain $\left(\Omega^{F E}\right.$ and $\left.\Omega^{P}\right)$, respectively. Note that for the dynamic scheme, the displacements $\mathbf{u}^{i}, i=F E$ or $P$ in the fifth term on the LHS of equation (111) and (112) are replaced by their accelerations $\ddot{\mathbf{u}}^{i}, i=F E$ or $P$ to obtain the discrete equations. The test and trial functions in a Bubnov-Galerkin method are approximated via the same shape function

$$
\begin{aligned}
\delta \mathbf{u}_{h}^{i}(\mathbf{X}, t) & =\sum_{J=1}^{N} N_{J}^{i}(\mathbf{X}, t) \delta \mathbf{u}_{J}^{i}(t) \\
\mathbf{u}_{h}^{i}(\mathbf{X}, t) & =\sum_{J=1}^{N} N_{J}^{i}(\mathbf{X}, t) \mathbf{u}_{J}^{i}(t)
\end{aligned}
$$

where the superscript $i$ indicates the corresponding domain $\left(\Omega^{F E}\right.$ and $\left.\Omega^{P}\right)$. The Lagrange multipliers are approximated to

$$
\begin{gathered}
\boldsymbol{\lambda}_{h}^{i}=\sum_{J=1}^{N} \Lambda_{J} \boldsymbol{\lambda}_{J}^{i} \\
\delta \boldsymbol{\lambda}_{h}^{i}=\sum_{J=1}^{N} \Lambda_{J} \delta \boldsymbol{\lambda}_{J}^{i}
\end{gathered}
$$


where for the $\Lambda$ the FE shape functions are chosen. Finally we obtain with the traction and displacement continuity the equation of motion

$$
\sum_{J=1} \mathbf{m}_{I J} \ddot{\mathbf{u}}_{J}=\mathbf{f}_{I}^{e x t}-\mathbf{f}_{I}^{\text {int }}
$$

with

$$
\begin{gathered}
\mathbf{m}=\left[\begin{array}{ccc}
\mathbf{m}^{F E} & 0 & -\mathbf{G}^{F E} \\
0 & \mathbf{m}^{P} & \mathbf{G}^{P} \\
-\mathbf{G}^{F E} & \mathbf{G}^{P} & 0
\end{array}\right] \\
\mathbf{u}=\left[\begin{array}{c}
\mathbf{u}^{F E} \\
\mathbf{u}^{P} \\
\boldsymbol{\lambda}
\end{array}\right] \\
\mathbf{F}=\left[\begin{array}{c}
\mathbf{f}^{i n t, 1}-\mathbf{f}^{\text {ext }, 1} \\
\mathbf{f}^{\text {int }, 2}-\mathbf{f}^{\text {ext }, 2} \\
-\mathbf{g}
\end{array}\right]
\end{gathered}
$$

and

$$
\begin{gathered}
\mathbf{m}_{I J}^{i}=\sum_{I} \int_{\Omega_{0}^{i}} \varrho_{0}^{i} N_{I}^{i} N_{J}^{i} d \Omega_{0}^{i}, i=F E \text { or } P \\
\mathbf{G}_{I J}^{i}=\int_{\Gamma_{0}^{u}} N_{I}^{i} \boldsymbol{\Lambda}_{J} d \Gamma_{0}^{i} \\
\mathbf{f}_{I}^{i n t, i}=\int_{\Omega_{0}^{i}} \nabla N_{I}^{i} \cdot \mathbf{P}^{i} d \Omega_{0}^{i}, i=F E \text { or } P \\
\mathbf{f}_{I}^{e x t, i}=\int_{\Gamma_{0}^{i}} \varrho_{0}^{i} N_{I}^{i} \mathbf{b}^{i}+N_{I}^{i} \overline{\mathbf{t}}^{i} d \Gamma_{0}^{i}, i=F E \text { or } P
\end{gathered}
$$

where the superscript $i$ designates either the particle or FE domain. Note that we used here a consistent mass matrix. When the mass is lumped, a scheme as explained in detail in table I can be used.

\subsection{Hybrid approximation}

The principle of the hybrid (finite element-meshfree method) coupling is explained in figure

7. For the particle approximation, the finite element domain is included in the sums of the meshfree approximation:

$$
\begin{gathered}
u_{I}^{h}(\mathbf{X})=\sum_{J} u_{J} N\left(\mathbf{X}_{J}-\mathbf{X}_{I}, h_{0}\right)+\int_{\Omega^{F E \cap P}} u^{F E} N\left(\mathbf{X}^{F E}-\mathbf{X}_{I}, h_{0}\right) d \Omega_{0} \\
\nabla u_{I}^{h}(\mathbf{X})=\sum_{J} u_{J} \nabla N\left(\mathbf{X}_{J}-\mathbf{X}_{I}, h_{0}\right)+\int_{\Omega^{F E \cap P}} u^{F E} \nabla N\left(\mathbf{X}^{F E}-\mathbf{X}_{I}, h_{0}\right) d \Omega_{0}
\end{gathered}
$$




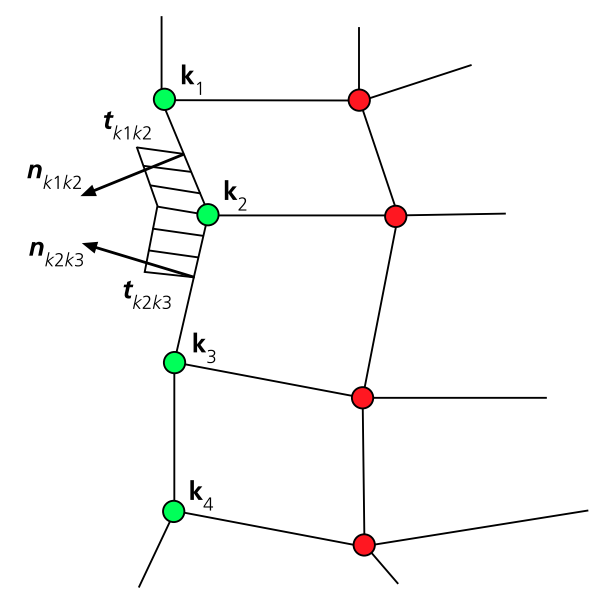

Figure 7. Hybrid Finite Element-Particle coupling

where the first term on the RHS of eq. (125) and (126) is the usual discrete meshfree approximation and the second term is the enhancement in the hybrid domain $\Omega^{F E} \cap P$. Applying this procedure to the typical SPH-form of the momentum equation, we obtain:

$$
\begin{gathered}
\ddot{\mathbf{u}}_{I}(\mathbf{X})=-\frac{1}{\varrho_{0}} \sum_{J}\left(\mathbf{P}\left(\mathbf{X}_{J}\right)+\mathbf{P}\left(\mathbf{X}_{I}\right)\right) \cdot \nabla N\left(\mathbf{X}_{J}-\mathbf{X}_{I}, h_{0}\right) \\
-\frac{1}{\varrho_{0}} \int_{\Omega^{F E \cap P}}\left(\mathbf{P}\left(\mathbf{X}^{F E}\right)+\mathbf{P}\left(\mathbf{X}_{I}\right)\right) \cdot \nabla N\left(\mathbf{X}_{J}-\mathbf{X}_{I}, h_{0}\right) d \Omega_{0}
\end{gathered}
$$

The incremental deformation gradient $\nabla \mathbf{u}\left(\mathbf{X}_{I}\right)$ is computed by the same technique:

$$
\begin{array}{r}
\nabla \mathbf{u}\left(\mathbf{X}_{I}\right)=-\sum_{J}\left(\mathbf{u}\left(\mathbf{X}_{J}\right)-\mathbf{u}\left(\mathbf{X}_{I}\right)\right) \otimes \nabla N\left(\mathbf{X}_{J}-\mathbf{X}_{I}, h_{0}\right) \\
-\int_{\Omega^{F E \cap P}}\left(\mathbf{u}\left(\mathbf{X}^{F E}\right)-\mathbf{u}\left(\mathbf{X}_{I}\right)\right) \otimes \nabla N\left(\mathbf{X}^{F E}-\mathbf{X}_{I}, h_{0}\right) d \Omega_{0}
\end{array}
$$

Especially the possibility to compute the deformation gradient is the large advantage of the hybrid coupling versus other strong - form couplings developed by Johnson [24] and Attaway et al. [2]. Since the particle sums remain symmetric, it is guaranteed that the deformation gradient is computed correctly. Another advantage is that the approximation is independent of the relation between the particle distance and the finite element length. Since no transition elements are needed for this coupling, adaptivity can be incorporated quite easily as proposed by Sauer [45].

The approach shown above can be easily extended to improved SPH approximation schemes, as normalized SPH (Sauer [45]) or stress point integration versions of the EFG method. For the finite elements, the integrals in the hybrid domain $\Omega^{F E \cap P}$ can be evaluated by Gauss quadrature. However, as shown by Sauer [45], using (in local coordinates) equally-spaced integration points in approximately the same density as the SPH particles gave the most accurate results for most examples tested. For a two dimensional quadrilateral element, a 
function in the discrete form can be computed as

$$
\int_{\Omega^{F E \cap P}} u(x, y) N(x, y) d \Omega_{0}=\sum_{J} \sum_{i=1}^{m} \sum_{j=1}^{n} w_{i} w_{j} u\left(\xi_{i}, \eta_{j}\right) N\left(x_{i}, y_{i}\right)\left|\mathbf{J}\left(\xi_{i}, \eta_{j}\right)\right|
$$

and its spatial derivative as

$$
\int_{\Omega^{F E} \cap P} u(x, y) \nabla N(x, y) d \Omega_{0}=\sum_{J} \sum_{i=1}^{m} \sum_{j=1}^{n} w_{i} w_{j} u\left(\xi_{i}, \eta_{j}\right) \nabla N\left(x_{i}, y_{i}\right)\left|\mathbf{J}\left(\xi_{i}, \eta_{j}\right)\right|
$$

where $w_{i}$ and $w_{j}$ are the weights in the corresponding direction $\xi_{i}$ and $\eta_{j}$, respectively, $\xi_{i}$ and $\eta_{j}$ are local coordinates, $m$ is the number of integration points in $\xi$-direction, $n$ the number of integration points in $\eta$-direction and $\mathbf{J}$ designates the Jacobian matrix.

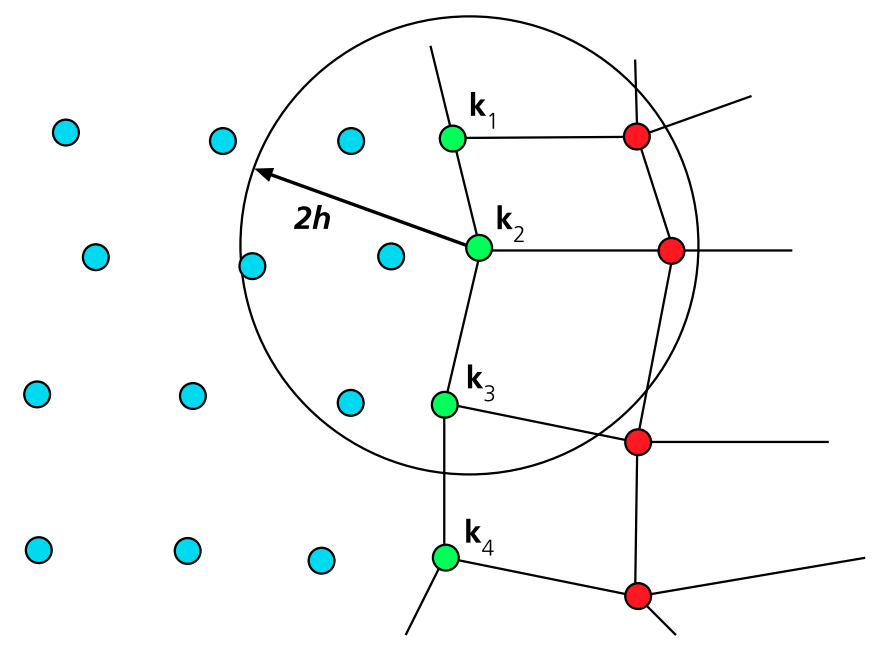

Figure 8. Force transmission on the element nodes in the hybrid domain

Crucial is how to apply the forces on the element nodes in the hybrid domain. Sauer [45] proposed two different techniques. One possibility is to impose the forces from the particle domain on the element-particle boundary as shown in figure 8. The stresses for boundary particle $k_{2}$ (see figure 8) are then:

$$
\mathbf{P}_{k_{2}}=\frac{\sum_{J} \mathbf{P}\left(\mathbf{X}_{J}\right) N\left(\mathbf{X}_{J}-\mathbf{X}_{I}, h_{0}\right)}{\sum_{J} N\left(\mathbf{X}_{J}-\mathbf{X}_{I}, h_{0}\right)}
$$

The traction on the adjacent element sides according to figure 8 can then be expressed as

$$
\begin{aligned}
& \mathbf{t}_{k_{1} k_{2}}=\mathbf{n}_{k_{1} k_{2}} \cdot \mathbf{P}_{k_{2}} \\
& \mathbf{t}_{k_{2} k_{3}}=\mathbf{n}_{k_{2} k_{3}} \cdot \mathbf{P}_{k_{2}}
\end{aligned}
$$


and the internal forces for node 2 :

$$
\mathbf{F}_{2}=\frac{l_{12}}{2} \mathbf{t}_{k_{1} k_{2}}+\frac{l_{23}}{2} \mathbf{t}_{k_{2} k_{3}}
$$

where $l_{12}$ denotes the length of the side from node $k_{1}$ to node $k_{2}$ and $l_{23}$ denotes the length of the side from node $k_{2}$ to node $k_{3}$. Care has to be taken at edges as shown in figure 9 . Therefore, a visibility criterion is incorporated, so that only particles contribute to the sums which can be seen by the corresponding element boundary node. In the following this coupling will be denoted as $H A-S$ coupling.

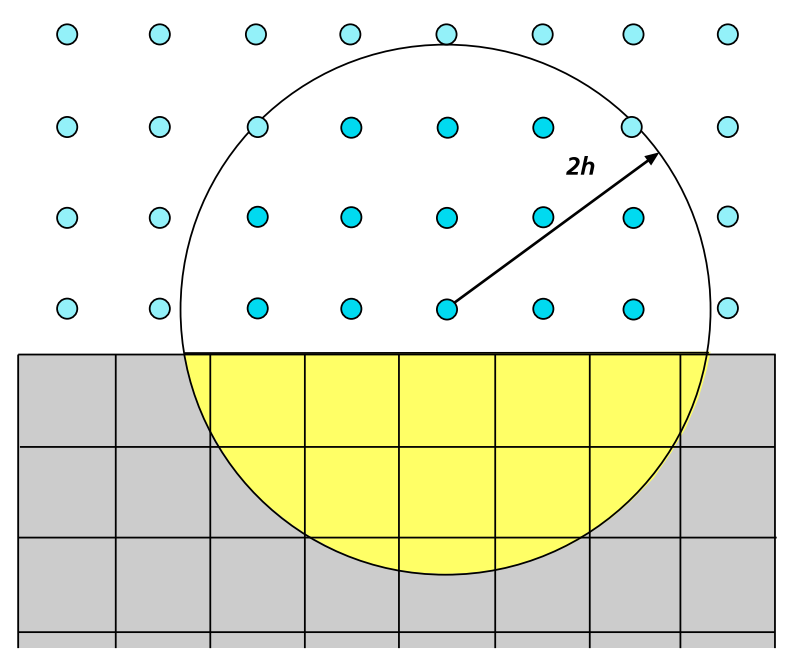

Figure 9. Edge to edge coupling using the visibility criterion

Another possibility to apply the forces from the particles to the finite elements, is shown in figure 10. The advantage of this methods is its robustness since it is not sensitive with respect to changes in the element topology and therefore easy to use in $3 \mathrm{D}$. The internal force can be calculated as

$$
\mathbf{F}=-w_{G P} \operatorname{det} \mathbf{J}_{G P}\left(\mathbf{P}\left(\mathbf{X}_{G P}^{F E}\right)+\mathbf{P}\left(\mathbf{X}_{I}\right)\right) \cdot N\left(\mathbf{X}^{F E}-\mathbf{X}_{I}, h_{0}\right)
$$

In the following, this coupling approach will be denoted as $H A-F$ coupling. We used the central difference time integration. 


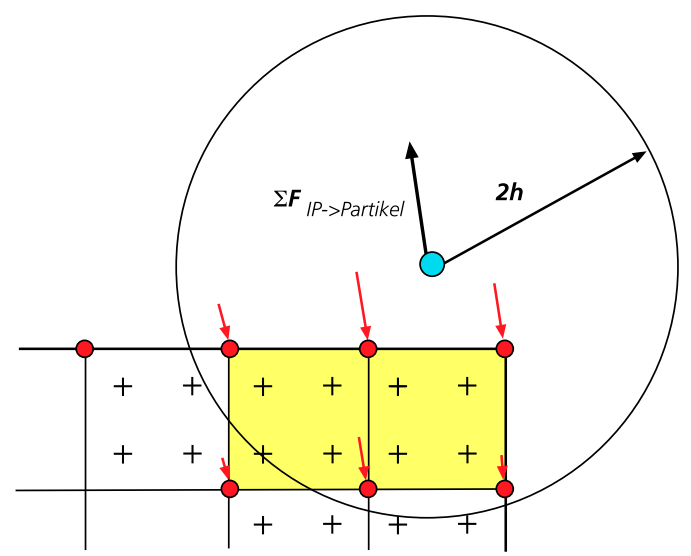

- particle

- node with interaction

mesh boundary

element with integration

points

sum of forces from

interaction with

elements

$\longrightarrow$ nodal reaction forces

Figure 10. Force transmission via interpolation 


\section{NUMERICAL EXAMPLES}

6.1. Static examples

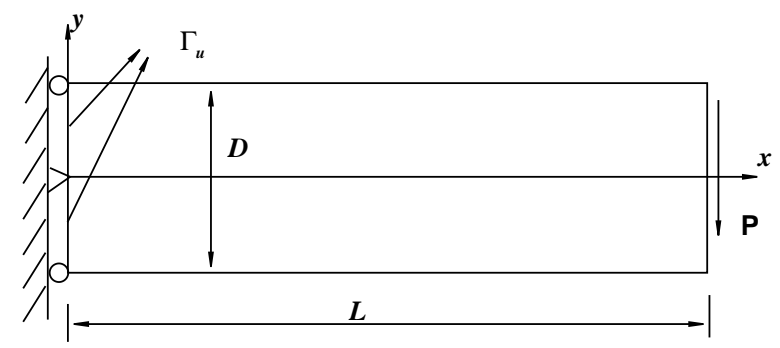

Figure 11. Timoshenko elastic beam

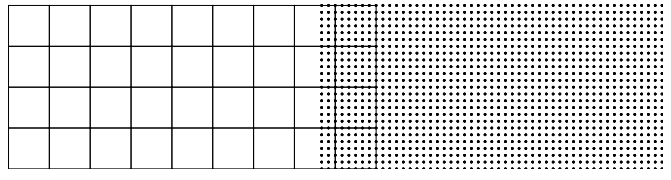

Figure 12. Bridging Domain Coupling: finite element and particle arrangement

6.1.1. Timoshenko Beam Problem We will test the different methods for a cantilever beam of length $L$ and height $D$ with a traction of $P$ at the free end as shown in figure 11 . The model is considered to be of unit thickness and in a state of plane stress. The exact solution of this problem has been given by Timoshenko [47]:

$$
\begin{aligned}
& u_{x}=-\frac{P}{6 E I}\left[(6 L-3 x) x+(2+\nu)\left(y^{2}-\frac{D^{2}}{4}\right)\right] \\
& u_{y}=\frac{P}{6 E I}\left[3 \nu y^{2}(L-x)+(4+5 \nu) \frac{D x^{2}}{4}+(3 L-x) x^{2}\right]
\end{aligned}
$$

where the moment of inertia $I=\frac{D^{3}}{12}$ and the other parameters used here are:

$$
E=3 \times 10^{7} \quad \nu=0.3 \quad D=12 \quad L=48 \quad P=1000
$$

The $L_{2}$ error displacement norm is checked in this example

$$
L_{2}=\frac{\left\|\mathbf{u}_{h}-\mathbf{u}_{\text {analytic }}\right\|}{\left\|\mathbf{u}_{\text {analytic }}\right\|}
$$

The coupling domain for the Bridging domain coupling in the initial configuration is shown in figure 12. For the other approaches the particle and the FE-domain do not overlap. 
The different coupling methods in section 5 are studied. Figure 13 shows the error in the displacement norm versus the element length. The best results are obtained from the Bridging domain coupling, the edge-to-edge coupling and the compatibility coupling. The differences are marginal. The coupling via ramp functions give similar results in the convergence rate though the absolute error is higher. The worst results are obtained by the master slave coupling and the hybrid coupling method.

As can be seen from figure 13 (see also table II), the convergence rate is decreasing with increasing refinement. Table II lists the values of the convergence rate for the different refinement steps.

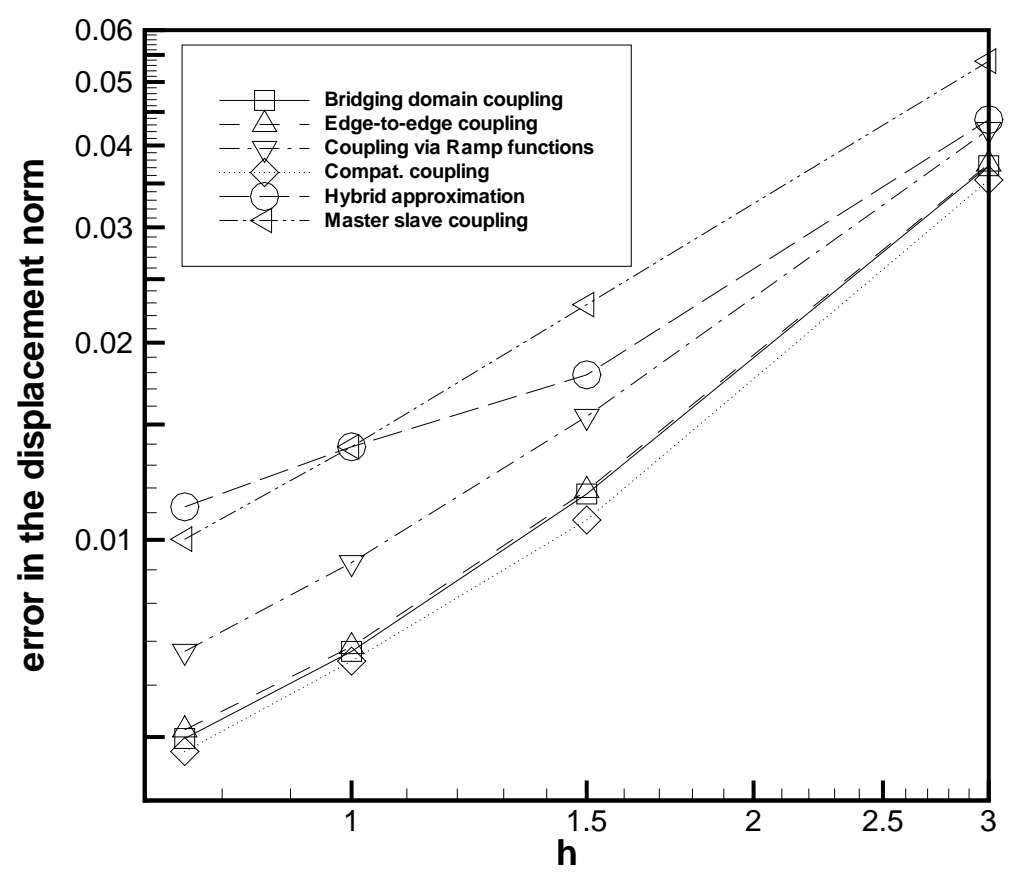

Figure 13. Error in the displacement norm for the different coupling methods for the static Timoshenko beam

6.1.2. Crack propagation problem The next example is a crack propagation problem in concrete. Therefore, a notched beam is loaded as shown in figure 14. The beam fails due to a mixed mode failure. Experimental data is available, see Arrea and Ingraffea [1]. Since particles have advantages over finite elements in crack problems, the area around the notch, where we expect the crack to propagate, is discretized with particles while the rest is discretized with a much coarser finite element mesh. We have also run computations with a pure meshfree discretization. The advantage of the hybrid scheme is the lower computational cost. 


\begin{tabular}{l|l|l|l}
\hline & $h_{1}-h_{2}$ & $h_{2}-h_{3}$ & $h_{3}-h_{4}$ \\
\hline & $3-1.5$ & $1.5-1.0$ & $1.0-0.75$ \\
\hline Bridging domain coupling & 1.66 & 1.365 & 1.06 \\
Edge-to-Edge Coupling & 1.657 & 1.354 & 1.02 \\
Coupling via ramp function & 1.455 & 1.267 & 1.03 \\
Comp. coupling & 1.72 & 1.23 & 1.1 \\
Master slave coupling & 1.23 & 1.23 & 1.13 \\
Hybrid coupling & 1.23 & 1.23 & 1.13 \\
\hline
\end{tabular}

Table II. Convergence rates

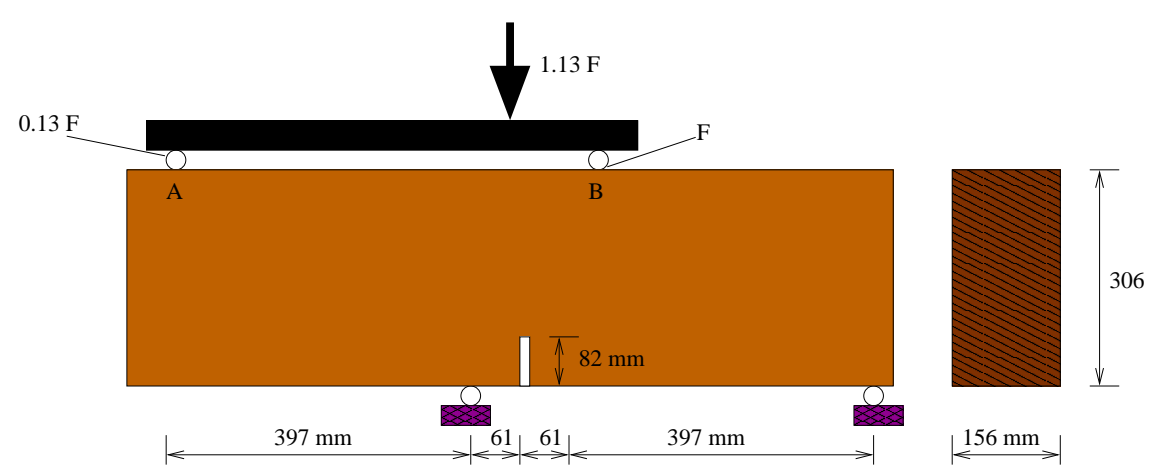

Figure 14. Test set up of the notched concrete beam of Arrea and Ingraffea [1]

The results in the crack path are pretty similar for all tested methods. We will show results for the coupling with Lagrange multipliers, the coupling with ramp functions and the master slave coupling. A typical discretization in the initial configuration is shown in figure 15. Figure 16 shows the discretization close to the transition between finite elements and particles for the case when particles are located directly on finite element nodes. We will compare the crack pathes and the load-displacement (right of the notch) curves to the experimental data.

We have chosen a combined continuum-discontinuum approach to describe the concrete, see Belytschko et al. [12]. The concrete is modelled with linear elastic material behavior in compression and with the Lemaitre model [29] in tension. In the discontinuous region, a linear traction-crack opening relation is applied. Details of this model can be found in Belytschko et al. [12].

For all hybrid discretization, we will show results for approximately 3500 particles in the region where the crack is expected. The adjacent region is modelled with 2700 elements. Finer meshes gave similar results.

For the pure EFG approximation, the total number of particles is approximately 13, 400. The crack pathes for the different coupled methods and the pure EFG discretization are shown in figure 17. The results look very similar but the pure meshfree discretization is computationally more expensive. A slightly curved crack can be observed which matches well with the experimental results. 


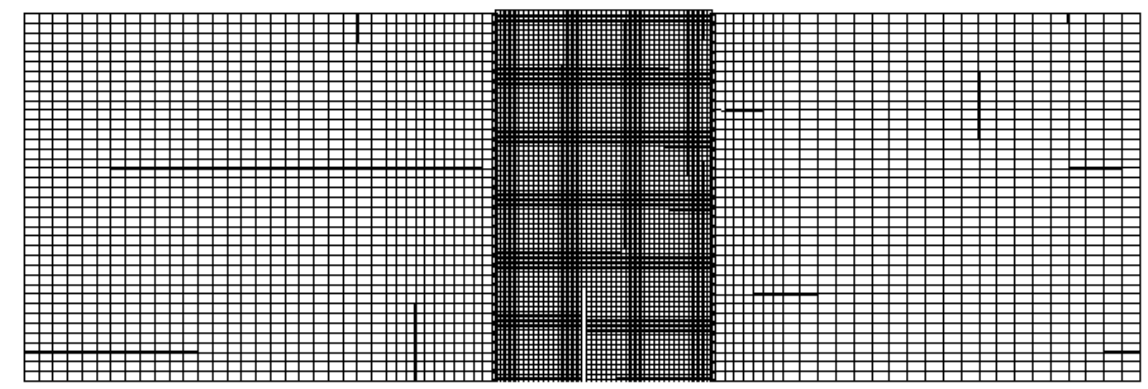

Figure 15. Discretization of the notched concrete beam of Arrea and Ingraffea [1]

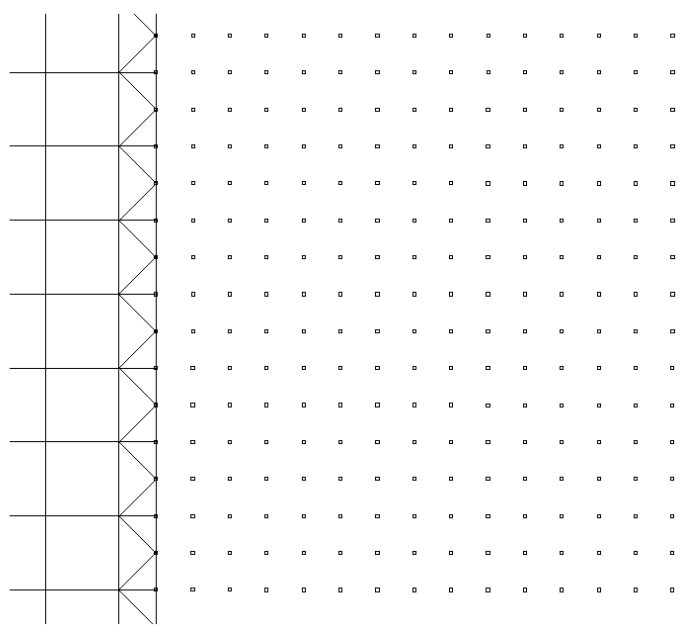

Figure 16. Discretization of the notched concrete beam of Arrea and Ingraffea [1]

The load-displacement (right of the notch) curves are shown in figure 18. All curves are lying within the experimental scatter and show marginal differences.

\subsection{Dynamic examples}

6.2.1. The rod with initial boundary velocity condition To investigate the coupling methods for dynamic problems, we consider a linear elastic rod. The rod is discretized in 2D (plane strain). It is $60 \mathrm{~mm}$ long and its height is $5 \mathrm{~mm}$. An initial velocity of $v=e^{\left(-\alpha(x-30)^{2}\right)}, \alpha=$ 0.025 is prescribed. The sound speed is $c=\sqrt{E / \varrho}$ with $E=210,000 M P a$ and $\varrho=$ $0.0078 \mathrm{~g} / \mathrm{mm}^{3}$, so the wave returns to its original position at $t=0.011563 \mathrm{~ms}$. This example was studied in [43] for pure particle methods. Xiao [51] showed in one dimension that the bridging domain coupling is superior to other coupling methods in wave reflection problems since spurious wave reflections are removed. 


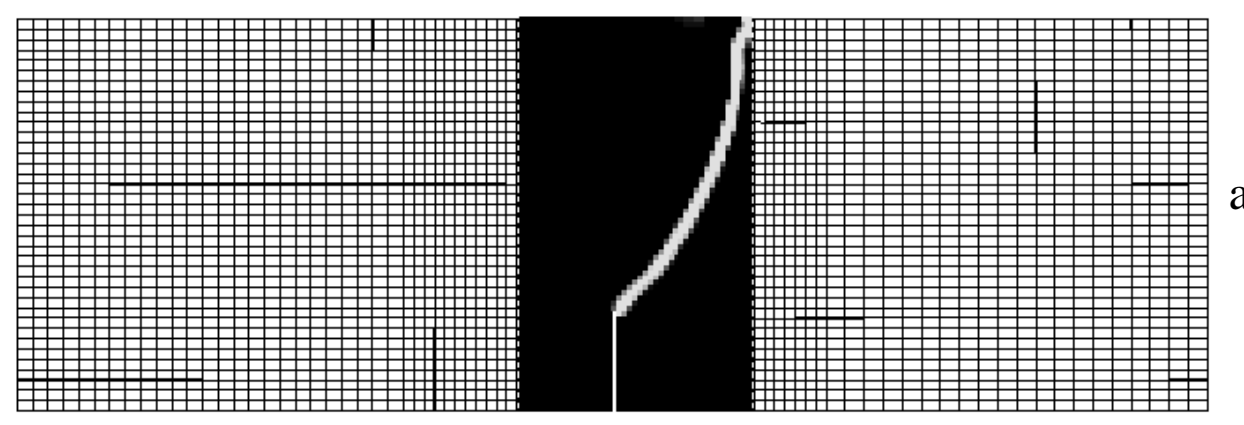

a)
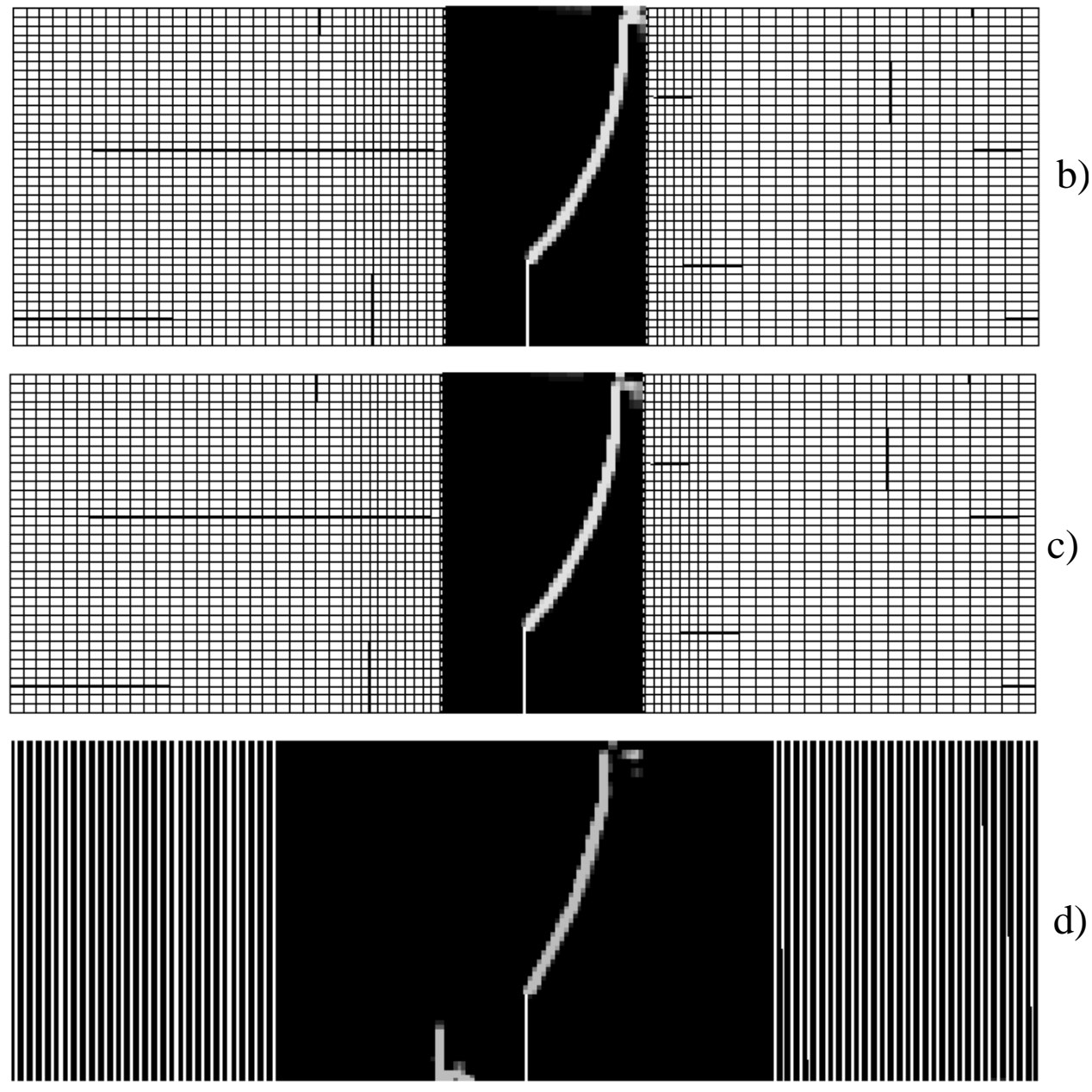

d)

Figure 17. Crack pathes for the hybrid coupling, a) rigid coupling, b) compatability coupling, c) coupling via ramp functions, d) pure meshfree discretization 


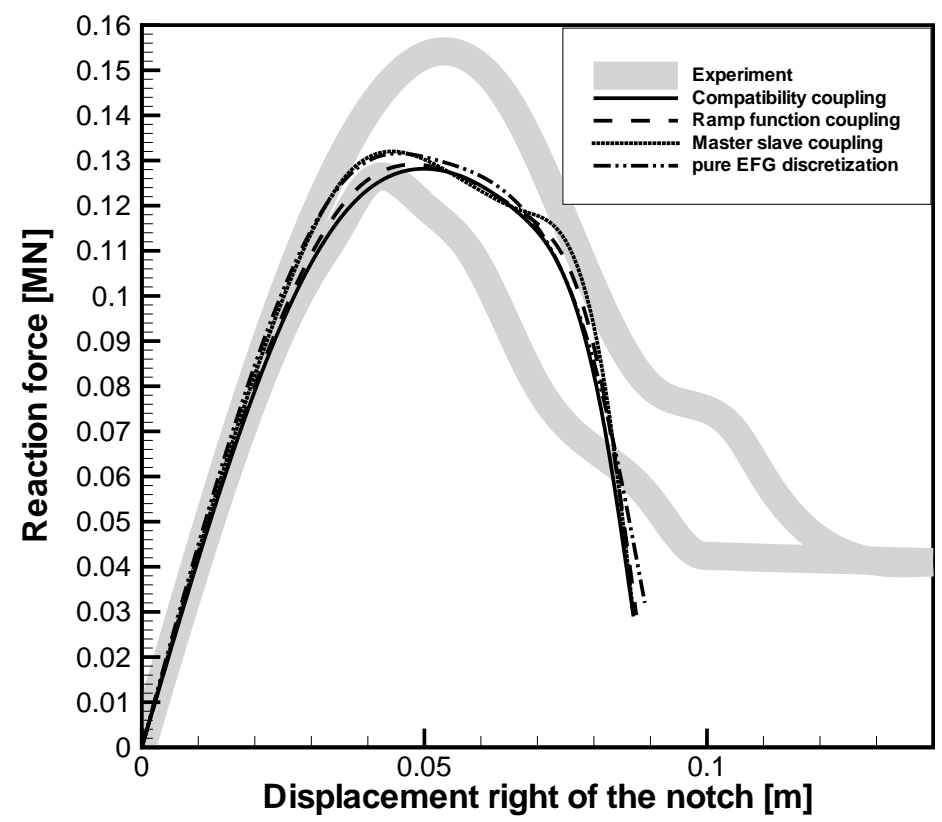

Figure 18. Load displacement curve of the different computations compared to the experimental data

We study different ratios between particles and finite elements nodes, from 1:1 up to 8:1. We also study the influence of the refinement. Half of the rod is discretized meshfree while the other part is discretized with finite elements as it was done in section 6.1.1 for the Timoshenko beam. The Young's modulus $E$ is set to 210,000 and the Poisson's ratio is $\nu=0.0$. The $L_{2}$ error in the velocities is given before and after the wave reflection:

$$
\|\operatorname{err}\|_{L_{2}}=\frac{\left\|\mathbf{v}^{h}-\mathbf{v}_{\text {analytic }}\right\|}{\left\|\mathbf{v}_{\text {analytic }}\right\|} .
$$

Additional to the coupling methods, we will give also the results for a pure meshfree discretization, i.e. EFG with stress point integration. In table III, the results are presented for approximately 20,000 nodes+particles and a ratio of 4:1. The error in the velocity is given for the different methods at two times. At $t=0.011563 \mathrm{~ms}$, the wave first reaches its original position, at $t=0.023126 \mathrm{~ms}$ it reaches its original position for the second time.

As can be seen, the best results are obtained by the pure meshfree discretization. Of course, there is no noise caused due to any coupling. The smallest error is obtained with the bridging domain coupling followed by the hybrid coupling method. The largest error occurs for the rigid coupling. Except for the pure meshfree discretization and the bridging domain coupling, it can seen that the error increases in time. This increase in error is probably caused by spurious 
Table III. $L_{2}$ error in the velocities for an initial condition (Gauss distribution of the velocities) for different particle methods before and after the wave reflection

\begin{tabular}{|c|c|c|c|}
\hline & $\begin{array}{c}\text { error at } \\
0.0116 \mathrm{~ms}\end{array}$ & $\begin{array}{c}\text { error at } \\
0.0231 \mathrm{~ms}\end{array}$ & $\begin{array}{c}\text { CPU time } \\
\text { [minutes] }\end{array}$ \\
\hline Master slave coupling & 0.0289 & 0.0465 & 5.3 \\
Hybrid coupling & 0.0141 & 0.0152 & 5.5 \\
Coupling via ramp functions & 0.0179 & 0.0323 & 5.5 \\
Comp. coupling & 0.016 & 0.03201 & 5.6 \\
Bridging domain coupling & 0.0107 & 0.0111 & 5.8 \\
EFG & 0.007842 & 0.007901 & 9.1 \\
\hline
\end{tabular}

wave reflections. Table III gives also the computation times for the different methods. As can be seen, the meshfree method gives the most accurate results but the computation time is approximately 2 times longer. It should be mentioned that half of the rod is discretized with particles, so that a dramatic speed up is expected if a smaller fraction of the structure is discretized with particles.

If the ratio between particles and finite element nodes is decreased, the error over time in the velocity norm increases in the hybrid method up to a factor of 2 for the tested example and a ratio of 1:1. However, for larger ratios, the hybrid method gives more accurate results than all the other methods tested (except for the bridging domain coupling). The bridging domain coupling gives also a nearly constant error at the two different times for lower ratios.

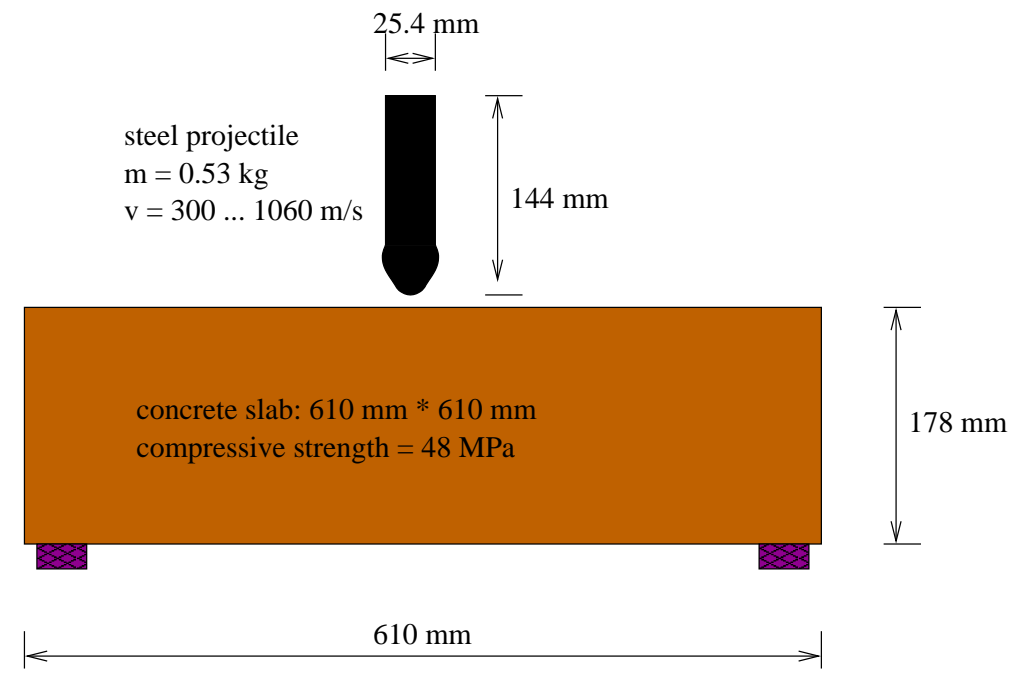

Figure 19. Experimental setup of the impact experiments preformed by Hanchak et al. [17]

6.2.2. Impact of a concrete slab Hanchak et al. [17] performed different impact experiments of reinforced concrete slabs. In their experiments, the concrete had a compressive strength of 
$48 M P A$ and 140 MPA, respectively. They used a $0.53 \mathrm{~kg}$ steel projectile which was shot onto a $0.178 m$ thick $0.61 m \times 0.61 m$ concrete slab, see figure 19 . Besides the initial velocity of the steel projectile they also measured its discharge velocity. The influence of the reinforcement $(5.7 \mathrm{~mm}$ diameter, $\mathrm{e}=76 \mathrm{~mm})$ in respect to the perforation resistance is negligible according to Hanchak et al. [17]. Interesting is the observation, that the concrete with a strength of 140 $M P a$ was able to increase the perforation resistance only slightly in respect to the concrete with a compressive strength of $48 \mathrm{MPa}$. The experimental setup is shown in figure 19.

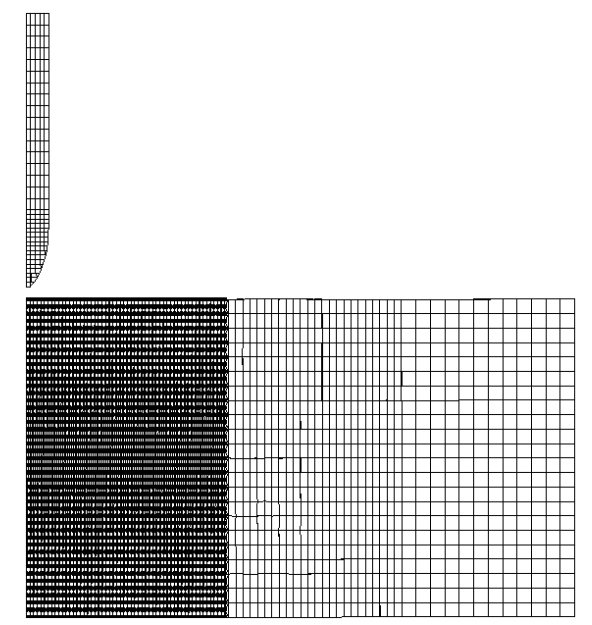

Figure 20. Hybrid discretization of the concrete slab

The particle and finite element discretization is examplarily shown for the ramp function coupling, figure 20. The region where we expect large deformations and a high damage is discretized with particles, the rest is discretized with finite elements. We made use of the symmetry and discretized only half of the projectile and the slab. At a discretization of 2520 particles and 836 elements for the concrete, we obtained mesh independent results which are presented here. The projectile is modelled with a pure FE mesh using 70 elements.

The reinforcement is modelled via an elastoplastic material law with a strain based failure. Simplified, a rigid bond between concrete and the reinforcement is assumed. For the concrete a constitutive model as described in Rabczuk et al. [42] was used. The initial elasticity modulus for concrete accounts for 36,000 $\mathrm{MPa}$ and the compressive strength for $48 \mathrm{MPA}$. All other material parameters can be found in [42].

As mentioned above, additionally to the experimental data, we compare our results to a pure meshfree (EFG) discretization.

Figure 21 shows the deformed concrete plate at different time steps for the two-dimensional simulation using the ramp function coupling method. Table IV compares the ejection velocity of the Hanchak experiments with different coupling methods and pure meshfree discretization. The coupling methods approximate the measured ejection velocity quite well for high impact velocities. For low impact velocities, larger discrepancies between the hybrid and pure meshfree approximation can be observed. We cannot explain why there are larger discrepancies for higher 


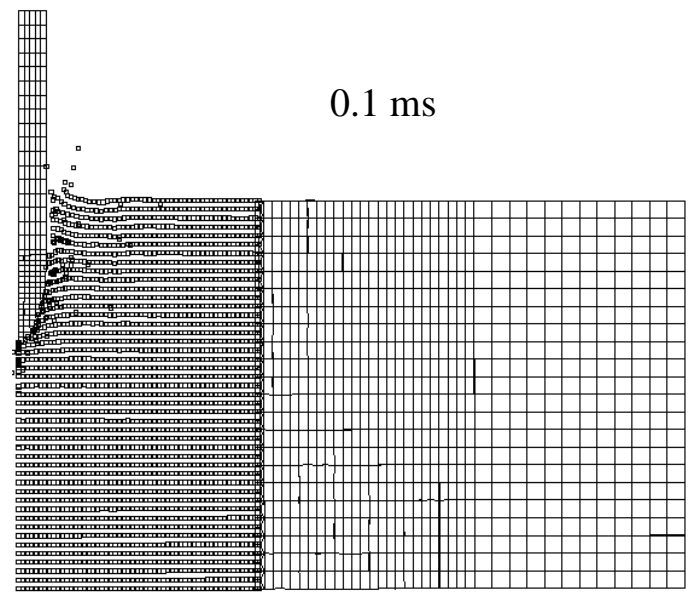

a)

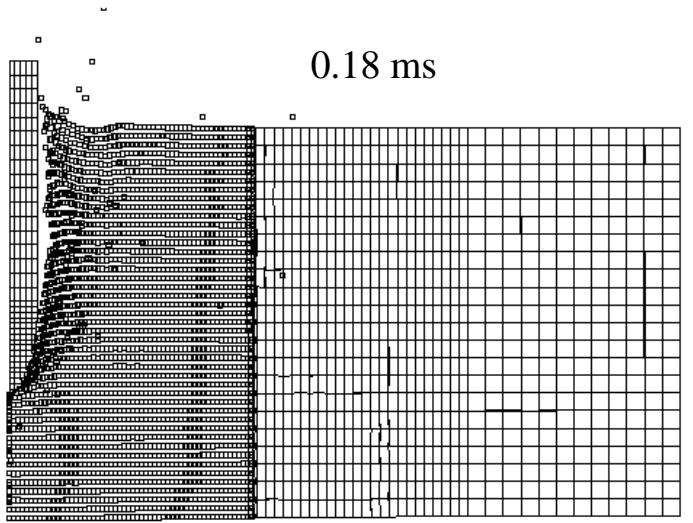

b)

Figure 21. Deformed configuration of the Hanchak slab using the ramp function coupling a) at 0.1 $\mathrm{ms}, \mathrm{b})$ at $0.18 \mathrm{~ms}$

Table IV. Ejection velocities for different impact velocities

\begin{tabular}{|c||c||c||c||c||c||c|}
\hline $\begin{array}{c}\text { Plate } \\
\text { (impact velocity) }\end{array}$ & experiment & $\begin{array}{c}\text { ramp function } \\
\text { coupling }\end{array}$ & $\begin{array}{c}\text { comp. } \\
\text { coupling }\end{array}$ & $\begin{array}{c}\text { hybrid } \\
\text { coupling }\end{array}$ & $\begin{array}{c}\text { master slave } \\
\text { coupling }\end{array}$ & $\begin{array}{c}\text { pure EFG } \\
\text { discretization }\end{array}$ \\
\hline $\mathrm{H} 1(360 \mathrm{~m} / \mathrm{s})$ & $67 \mathrm{~m} / \mathrm{s}$ & $12 \mathrm{~m} / \mathrm{s}$ & $22 \mathrm{~m} / \mathrm{s}$ & $69 \mathrm{~m} / \mathrm{s}$ & $26 \mathrm{~m} / \mathrm{s}$ & $76 \mathrm{~m} / \mathrm{s}$ \\
\hline $\mathrm{H} 2(430 \mathrm{~m} / \mathrm{s})$ & $214 \mathrm{~m} / \mathrm{s}$ & $175 \mathrm{~m} / \mathrm{s}$ & $184 \mathrm{~m} / \mathrm{s}$ & $207 \mathrm{~m} / \mathrm{s}$ & $183 \mathrm{~m} / \mathrm{s}$ & $216 \mathrm{~m} / \mathrm{s}$ \\
\hline $\mathrm{H} 3(750 \mathrm{~m} / \mathrm{s})$ & $615 \mathrm{~m} / \mathrm{s}$ & $604 \mathrm{~m} / \mathrm{s}$ & $611 \mathrm{~m} / \mathrm{s}$ & $616 \mathrm{~m} / \mathrm{s}$ & $611 \mathrm{~m} / \mathrm{s}$ & $620 \mathrm{~m} / \mathrm{s}$ \\
\hline $\mathrm{H} 4(1060 \mathrm{~m} / \mathrm{s})$ & $947 \mathrm{~m} / \mathrm{s}$ & $947 \mathrm{~m} / \mathrm{s}$ & $949 \mathrm{~m} / \mathrm{s}$ & $948 \mathrm{~m} / \mathrm{s}$ & $948 \mathrm{~m} / \mathrm{s}$ & $950 \mathrm{~m} / \mathrm{s}$ \\
\hline
\end{tabular}

impact velocities but we attribute these discrepancies to wave effects at the boundaries. The computation time for the pure meshfree discretization is approximately 8 times higher than for the coupling methods. The discrepancies in computational costs are marginal between the different methods.

\section{SUMMARY AND DISCUSSION}

We gave an overview about different particle-finite element coupling techniques; master slave couplings, coupling via ramp functions, compatibility coupling, bridging domain coupling and hybrid coupling.

Major theme is to use coupled methods due to computational efficiency. Local domains where cracks or large deformations are expected should be discretized with particles while other domains should be discretized with finite elements.

For the cantilever beam problem, we observe, that the master slave and the hybrid coupling gave the worst results. All other methods showed similar accuracy. All coupling methods gave 
similar results for the static Arrea-Ingraffea beam problem.

For the rod with initial boundary condition, only the bridging domain coupling and the hybrid coupling method gave a nearly constant error with time. In all other methods, the error increased with time that indicates spurious wave reflection. One drawback of the hybrid method is that the error over time in the velocity norm increases with decreasing ratio of particles to nodes at the interface. However, the hybrid method has the advantage that adaptivity can be incorporated quite easily compared to all other methods and is for large scale problems such as impacts probably most efficient. At the start, finite elements are used which can be transformed into finite elements. However, one can question if these methods are more accurate than remeshing algorithms since data has to be mapped as in remeshing finite element algorithms. Sauer [45] studied these issues and investigations are still underway.

The best choice for a coupling methods is definitely problem dependent. From the implementational point of view, the master slave coupling is probably the easiest and the bridging domain coupling is by far the most challenging one to code. For linear elasto-statics, we have observed that the hybrid method and the master slave coupling gave the worst results. However, when applying these methods to non-linear problems with local cracking, all coupling methods performed similarly well.

For wave propagation problems, only the bridging domain coupling and the hybrid coupling gave sufficient results. In all other methods, the error increased in time. Also comparisons with experimental data and pure meshfree discretizations indicate that spurious wave reflections influence the results.

Finally, we would like to mention that the different coupling techniques have some typical applications. While the bridging domain method was successfully applied in multiscale simulations -where often atoms were used instead of particles- as demonstrated by Xiao [51], the compatibility coupling e.g. was applied to static failure of reinforced concrete structures, [44]. Due to the wide application area, the limit of different numerical methods is not surprising. The development of new coupling method is still a hot topic of ongoing research.

\section{REFERENCES}

1. Arrea M., Ingraffea A.R.: Mixed-mode crack propagation in mortar and concrete, Rep. No. 81-13, Dept. of Struct. Eng., Cornell University Ithaka, N.Y., 1982

2. Attaway S.W., Heinstein M.W., Swegle J.W.: Coupling of Smoothed Particle Hydrodynamics with the Finite Element Method, Nuclear Engineering and Design 150, 1994, Post-SMIRT Impact IV Seminar Berlin

3. Babuska I., Melenk J.M.: The partition of unity finite element method, University of Maryland, Technical Note BN-1185, 1995

4. Belytschko T., Organ D., Krongauz Y.: A coupled finite element-element-free Galerkin method, Computational Mechanics 17 (1995), 186-195

5. Belytschko T. Krongauz Y., Dolbow J., Gerlach C.: On the completeness of Meshfree Particle methods, International Journal for numerical methods in Engineering 43 (1998), 785-819

6. Belytschko T., Xiao S.P.: A bridging domain method for coupling continua with molecular dynamics, Computer Methods in Applied Mechanics and Engineering, 193 (2004), 1645-1669

7. Belytschko T., Lu Y.Y., et al.: Element-free Galerkin methods, International Journal for Numerical Methods in Engineering, 37 (1994), 229-256

8. Belytschko T.: Crack propagation by element free Galerkin methods, Engineering Fracture mechanics, $51 / 2$ (1995), 295-315

9. Belytschko T., Lu Y.Y.: Element-free Galerkin methods for static and dynamic fracture, Int. J. Solids Strucutres 32 (1995), 2547-2570

10. Belytschko T., Liu W.K., Moran B.: Nonlinear Finite Elements for Continua and Structures, 2000, John Wiley and Sons Ltd., New York, USA 
11. Belytschko T., Xiao S.P.: Stability analysis of Particle Methods with Corrected Derivatives, Computers and Mathematics with Applications 43 (2000), 329-350

12. Rabczuk T., Belytschko T.: Cracking particles: A simplified meshfree method for arbitrary evolving cracks, International Journal for Numerical Methods in Engineering, Vol. 61 (13), 2316-2343, 2004

13. Chen J.S., Pan C., Wu C.T., Liu W.K.: Repdroducing kernel particle methods for large deformation analysis of nonlinear structures, Computer Methods in Applied Mechanics and Engineering, Vol. 139, 195-227, 1996

14. Chen J.S., Pan C., Roque, C.M.O.L., Wang H.P.: A Lagrangian Reproducing kernel particle method for metal forming analysis, Computational Mechanics, Vol. 22, 289-307, 1998

15. Duarte C.A., Oden J.T.: Hp clouds - a meshless method to solve boundary-value problems, TICAM Report 95-05, 1995

16. Gingold R.A., Monaghan J.J.: Smoothed particle hydro-dynamics: theory and applications to non-spherical stars, Mon. Not. R. astr. Soc., 181, 1977, 375-389

17. Hanchak S.J., Forrestal M.J., Young E.R., Erhrgott J.Q.: Perforation of concrete slabs with 48 MPa (7 ksi) and 140 MPA (20 ksi) unconfined compressive strengths, Int. J. Impact Eng., Vol. 12, 1992, 1-7

18. Han W., Wagner G.J., Liu W.K.: Convergence analysis f a hierarchical enrichment of dirichlet boundary conditions in a meshfree method, International Journal for Numerical Methods in Engineering, 2002, 53(6), 1323-1336

19. Haeusler-Combe U.: Elementfreie Galerkin-Verfahren, Grundlagen und Einsatzmoeglichkeiten, Habilitation, Institut fuer Massivbau und Baustofftechnologie, Universitaet Karlsruhe, 2001

20. Hao S., Liu W.K., Belytschko T.: Moving Particle Finite Element Method with Global smoothness, International Journal for Numerical Methods in Engineering, 2004, 59(7), 1007-1020

21. Hegen D.: Element free Galerkin methods in combination with finite element approaches, Computer Methods in applied Mechanics and Engineering, 1996, 135, 143-166

22. Huerta A., Fernandez-Mendez S.: Enrichment and coupling of the finite element and meshless method, Int. J. Numer. Meth. Engng. 2000, 48, 1615-1636

23. Huerta A., Fernandez-Mendez S., Liu W.K.: A comparison of two formulations to blend finite elements and mesh-free methods, Computer Methods in applied Mechanics and Engineering, 2004, 193 (12-14), 1105-1117

24. Johnson G.R.: Linking of Lagrangian Particle Methods to Standard Finite Element Methods for High Velocity Impact Copmutations, Nuclear Engineering and Design 150, 1994, Post-SMIRT Impact IV Seminar Berlin

25. Johnson G.R., Stryk R.A., Beissel S.R.: SPH for high velocity impact computations, Computer Methods in Applied Mechanics and Engineering, 1996, 139, 347-374

26. Kadowaki H., Liu W.K.: Bridging Multi-Scale Method for Localization Problems, Computer Methods in Applied Mechanics and Engineering, 2004, 193 (30-32), 3267-3302

27. Kadowaki H., Liu W.K.: A multiscale approach for the micropolar continuum model, Computer Modeling in Engineering and Sciences, 2005, 7(3), 269-282

28. Karutz H.: Adaptive Kopplung der Elementfreien Galerkin Methode mit der Methode der Finiten Elemente bei Rissfortschrittsproblemen, Dissertation, 2000, Institut fuer Statik und Dynamik der Ruhr Universitaet Bochum, VDI-Verlag, Reihe 18, Band 255

29. Lemaitre J.: Evaluation of dissipation and damage in metal submitted to dynamic loading, Proceedings ICM 1, 1971

30. Shaofan Li, Hongsheng Lu, Weimin Han, Wing Kam Liu and Daniel C. Simkins: Reproducing kernel element method Part II: Globally conforming Im/Cn hierarchies, Computer Methods in Applied Mechanics and Engineering, 2004, 193, 953-987

31. Li S., Liu W.K.: Meshfree and Particle Methods and Their applications, Applied Mechanics Review, 2002, $55,1-34$

32. Li S., Liu W.K.: Meshfree Particle Methods, Springer, 2004

33. Liu W.K., Chen Y.: Wavelet and Multiple Scale Reproducing kernel particle methods, International Journal for Numerical Methods in Fluid Dynamics, 1995, 21, 901-931

34. Liu W.K., Uras R.A., Chen Y.: Enrichment of the finite element method with reproducing kernel particle method, Journal of Applied Mechanics 1997, 135, 143-166

35. Liu W.K., Adee J., Jun S.: Reproducing kernel and wavelet particle methods for elastic and plastic problems, ADvances Computational Methods for Material Modeling, (Eds D.J. Benson and R.A. Asaro AMD 180 and PVP 268 ASME), pages 175-190, 1993

36. Liu W.K., Chen Y., Jun S., Chen S., Belytschko T., Pan C., Uras R.A., Chang C.T.: Overview and applications of the reproducing kernel particle methods, Archives of Computational Methods in Egnineering, State of the art reviews, 3:3-80, 1996

37. Liu W.K, Jun S., Adee J., Belytschko T.: Reproducing kernel particle method for structural dynamics, International Journal for Numerical Methods in Engineering, 38:1665-1679, 1995

38. Liu W.K., Jun S., Zhang Y.F.: Reproducing kernel partilce methods, International Journal for Numerical Methods in FLuid, 20:1081-1106, 1995 
39. Wing Kam Liu, Weimin Han, Hongsheng Lu, Shaofan Li and Jian Cao: Reproducing kernel element method. Part I: Theoretical formulation, Computer Methods in Applied Mechanics and Engineering, 2004, 193, 933951

40. Liu W.K., Karpov E.G., Zhang S., Park H.S.: An Introduction to Computational Nanomechanics and Materials, Computer Methods in Applied Mechanics and Engineering, 2004, 193, 1529-1578

41. Lucy: A numerical Approach to the Testing of Fission Hypothesis, Astronomical Journal, 82, 1977, 10131024

42. Rabczuk T., Eibl J.: Simulation of high velocity concrete fragmentation using SPH/MLSPH, Int. J. Numer. Meth. Engng, 2003, 56, 1421-1444

43. Rabczuk T., Belytschko T., Xiao S.P.: Stable particle methods based on Lagrangian kernelss, Computer Methods in Applied Mechanics and Engineering, 2004, 193, 1035-1063

44. Rabczuk T., Belytschko T.: Application of meshfree methods to static fracture of reinforced concrete structures, accepted in International Journal of Fracture

45. Sauer M.: Adaptive Koppling des netzfreien SPH-Verfahrens mit finiten Elementen zur Berechnung von Impaktvorgaengen, Dissertation, 2000, Universitaet der Bundeswehr Muenchen, Institut fuer Mechanik und Statik

46. Daniel C. Simkins, Jr., Shaofan Li, Hongsheng Lu and Wing Kam Liu: Reproducing kernel element method. Part IV: Globally compatible Cn (n1) triangular hierarchy, Computer Methods in Applied Mechanics and Engineering, 2004, 193, 1013-1034

47. Timoshenko S. P., Goodier J. N.: Theory of Elasticity, McGraw Hill, New York, 1970

48. Wagner G.J., Liu W.K.: Hierarchical Enrichment for Bridging Scales and Meshfree Boundary Conditions, International Journal for Numerical Methods in Engineering, 2001, 50, 507-524

49. Wagner G.J., Liu W.K.: Coupling of Atomic and Continuum Simulations using a bridging scale decomposition, Journal of Computational Physics, 2003, 190, 249-274

50. Xiao S.P., Belytschko T.: Material Stability Analysis of Particle Methods, submitted

51. Xiao S.P.: Atomistic Simulations of Nanotube Fracture and Stability analysis of particle methods, PhD thesis, 2002, Northwestern University, Department of Mechanical Engineering 